\title{
ARP-T1 is a ciliogenesis protein associated with a novel ciliopathy in inherited basal cell cancer, Bazex-Dupré-Christol Syndrome
}

Hyun-Sook Park ${ }^{1, \S}$, Eirini Papanastasi ${ }^{1}, \S$, Gabriela Blanchard ${ }^{1}$, Elena Chiticariu1, Daniel Bachmann ${ }^{1}$, Markus Plomann², Fanny Morice-Picard ${ }^{3}$, Pierre Vabres ${ }^{4}$, Asma Smahi ${ }^{5,6}$, Marcel Huber $^{1}$, Christine Pich ${ }^{1}$, and Daniel Hohl ${ }^{1, *}$

${ }^{1}$ Department of Dermatology, CHUV-FBM UNIL, Hôpital de Beaumont, Lausanne, Switzerland

${ }^{2}$ Center for Biochemistry, University of Cologne, D-50931, Cologne, Germany

$10{ }^{3}$ Department of Dermatology, CHU, Hôpital Pellegrin, Bordeaux, France

$11{ }^{4}$ Department of Dermatology, CHU, Hôpital du Bocage, Dijon, France

12 'Paris Descartes University, Sorbonne Paris Cité, Paris, France

${ }^{6}$ IMAGINE Institute INSERM UMR 1163, Paris, France

$\S$ These authors contributed equally to this work

*Lead Contact: daniel.hohl@chuv.ch

\section{ABSTRACT}

18 Actin-Related Protein-Testis1 (ARP-T1)/ ACTRT1 gene mutations cause the Bazex-Dupré19 Christol Syndrome (BDCS) characterized by follicular atrophoderma, hypotrichosis and basal cell cancer. Here, we report an ARP-T1 interactome (PXD016557) involved in ciliogenesis, endosomal recycling and septin ring formation. Consequently, ARP-T1 localizes to the midbody during cytokinesis and the basal body of primary cilia in $\mathrm{G}_{0}$. Tissue samples from BDCS patients show reduced ciliary length with significant correlations of ARP-T1 expression levels, confirmed by ACTRT1 knock down. We report that BDCS is a novel ciliopathy and the first case of a skin cancer ciliopathy, where ARP-T1 plays a critical role to prevent pathogenesis. 


\section{INTRODUCTION}

Basal cell cancer (BCC) of the skin is the most frequent human cancer. Bazex-Dupré-Christol Syndrome (BDCS) is an X-linked dominantly inherited syndrome form of BCC, without male-tomale transmission, affecting primarily the hair follicle (HF), resulting in the triad of hypotrichosis, follicular atrophoderma and BCC ${ }^{1-3}$. Hypotrichosis and follicular atrophoderma develop around birth and BCC early in adulthood. BDCS less frequently presents with milia, hypohidrosis, facial pigmentation and trichoepithelioma, and thus combines developmental cutaneous anomalies with tumor predisposition, i.e. an ectodermal dysplasia with a hereditary tumor syndrome ${ }^{4,5}$. Unlike most cases of BCC, which are sporadic, BDCS is an inherited syndromic form of BCC. The insertion mutation ACTRT1 547_548InsA, creating a shift of the reading frame, results in a truncated protein of 198 amino acids leading to a loss of function of the protein. ARP-T1 was also found depleted in families with germline mutations of non-coding sequences surrounding ACTRT1 postulated to belong to enhancers transcribed as non-coding RNAs. As these mutations segregate with the disease, ARP-T1 can be considered as a tumor suppressor in BDCS ${ }^{6}$. ARP-T1 was first isolated as a major acidic component of the cytoskeletal calyx from the head of bull spermatozoa. ARP-T1 is expressed specifically late in spermatid differentiation in testis, where it locates to the postacrosomal region and the centriole ${ }^{7}$, establishing a link to the primary cilium (PC) of epidermal cells implicated in BCC development.

Cilia are microtubule-based organelles that are either motile as in sperm propel or nonmotile (primary) acting as sensory antenna, receiving signals from other cells nearby. The sensory capacity of $\mathrm{PC}$ is founded on the spatio-temporal localization of diverse receptor complexes such as PTCH, RTKs, TGF $\beta$ R, NOTCH receptors, GPCRs including SMO, ion channels and extracellular matrix receptors along the cilium ${ }^{8}$. Ciliary communication is often compromised in cancer ${ }^{9}$, and faulty Hedgehog $(\mathrm{HH})$ signaling implicated prototypically in medulloblastoma and BCC. Indeed, BCC cells are frequently ciliated, and activated $\mathrm{HH}$ signaling within $\mathrm{PC}$ is a key driver in BCC pathogenesis ${ }^{10}$. BCCs generally show abnormal activation of the $\mathrm{HH}$ signaling pathway, ascribing its constitutive activation as a prerequisite for the tumor development ${ }^{11}$. $\mathrm{HH}$ signaling in its "off" state is characterized by PTCH1 repressing SMO activity. PC have a dual role and are required for or may repress tumor formation ${ }^{12,13}$, depending on the level of the molecular 56 interaction ${ }^{9}$.

PC assembly is a complex process emanating from the mother centriole with its appendages and differentiating upon cell cycle exit: the basal body, which nucleates the PC with apical CEP164 ${ }^{14}$, a component of distal appendages, EHD1/EHD3 and SNAP29 ${ }^{15}$ to initiate primary vesicle formation and ciliogenesis. Assembly of the PC includes recruitment of periciliary membranes,

61 transition zone components and a machinery of intraflagellar transport proteins to form the ciliary 62 vesicle and to set the base for formation of the microtubular ciliary axoneme ${ }^{15,16}$. The PC is separated from the cytosol by the basal body and anchored by transition fibers/distal appendages in the ciliary pocket or periciliary plasma membrane (PM) ${ }^{17}$. Transition fibers and transition zone with its $\mathrm{Y}$-shaped linkers serve as a ciliary gate for the cilio-cytoplasmic transport sharing some functional similarities with the nuclear pore complex ${ }^{17,18}$. Polarized membrane trafficking to the cilium and the vesicular transport machinery, which targets Golgi derived vesicles and apical recycling endosomes containing essential cargo, such as GPCRs, to the periciliary PM or the ciliary pocket depends on small GTPases, notably RAB8 and RAB11, Rabin 8 and the BBSome, a stable complex of seven proteins implicated in the ciliopathy of Bardet-Biedl Syndrome (BBS) ${ }^{8}$. Here, we demonstrate that ARP-T1 is a basal body protein and is involved in ciliogenesis by interacting with the ciliary machinery. Mutations in ACTRT1 or its enhancer elements, as found in the tumor samples of BDCS patients, as well as ACTRT1 knockdown give rise to the abnormally 
shortened cilia, and this may be caused by a disordered diffusion barrier. Ciliopathies encompass most human organ systems in eyes, nose, ears, organ placement, energy homeostasis, infertility, skeleton, reproductive system, brain, hydrocephalus, heart, chronic respiratory problems, kidney and liver ${ }^{19}$. We report that BDCS is the first ciliopathy of epidermal development and cancer.

\section{RESULTS}

ARP-T1 is expressed in differentiated human keratinocytes and human epithelial cells.

We examined the expression of ARP-T1 in NHEK (Fig. 1a,b) and HaCaT (Fig. 1c,d) cultured under differentiating conditions with high calcium up to seven days. The expression of ARP-T1 increased in a terminal differentiation dependent manner as indicated by the expression of Keratin 10 (K10) in differentiating keratinocytes at both mRNA (Fig. 1a,c) and protein levels (Fig. 1b,d). To investigate whether ARP-T1 expression was only induced in keratinocytes or whether it was related to differentiation, we also analyzed its expression in two differentiating retinal-pigmented epithelial (RPE) cells, ARPE-19 (Fig. 1e,f) and hTERT-RPE1 (Fig. 1g,h). These cells were differentiated under serum starvation for 35 days or $48 \mathrm{~h}$ respectively. During RPE differentiation, ciliogenesis is known to increase ${ }^{14,20,21}$ and IFT88 is a well-characterized protein involved in RPE ciliogenesis 22,23 . We assessed RPE differentiation monitoring with the expression of the ciliary protein IFT88 (Fig. 1f,h), and with the increased number of ciliated cells (Extended data Fig. 1a). Similar to keratinocytes, differentiated RPE cells exhibited an increased ARP-T1 expression after differentiation, at both mRNA (Fig. 1e,g) and protein levels (Fig. 1f,h, quantified in Extended data 94 Fig. 1b,c).
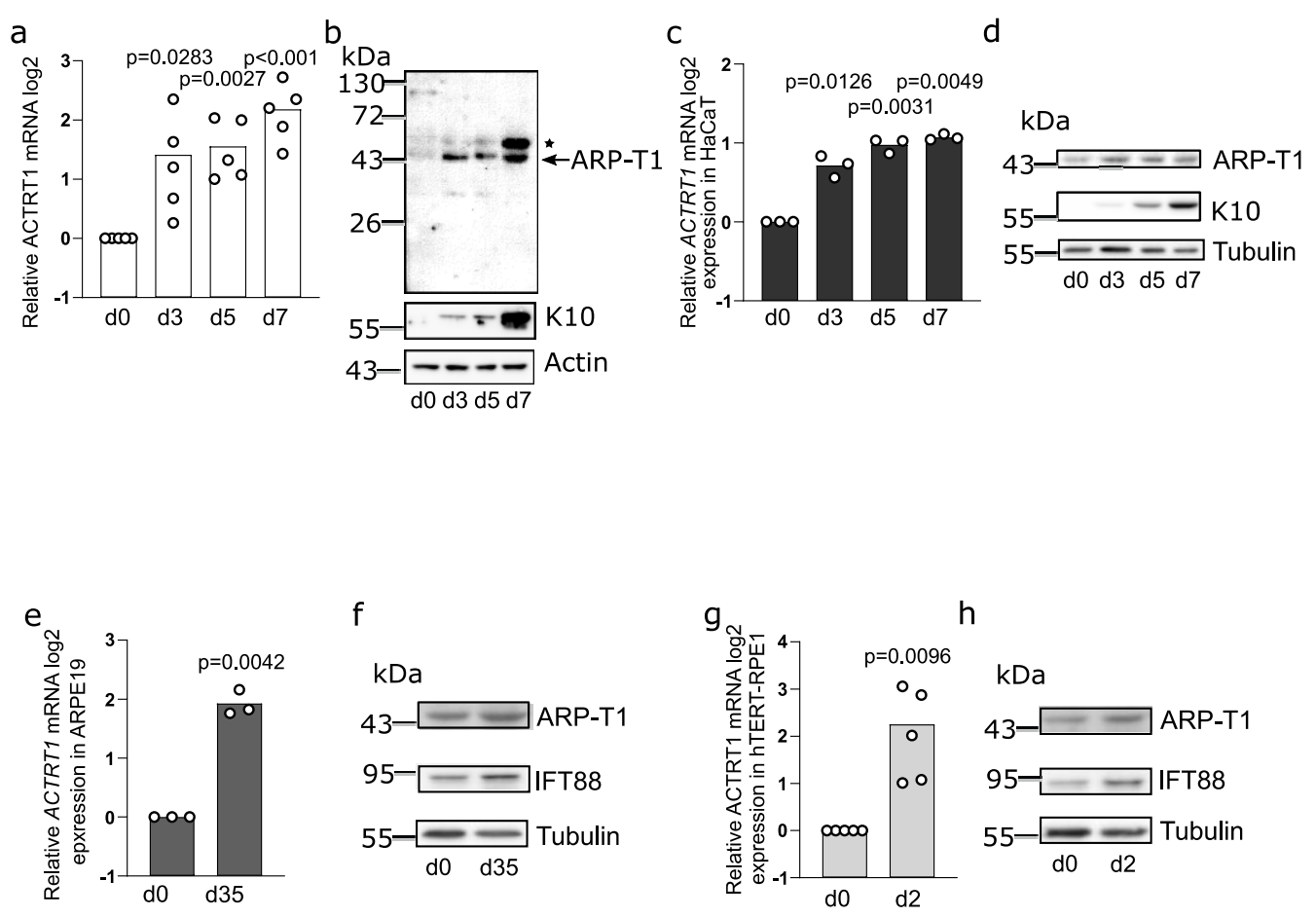


\section{ACTRT1 mRNA is regulated by non-canonical hedgehog pathway and protein kinase $\mathrm{C}$.}

ARP-T1 was previously reported to inhibit GLI1 expression and be involved in $\mathrm{HH}$ signaling ${ }^{6}$. We treated $\mathrm{HH}$ responsive hTERT-RPE1 cells with Smoothened (SMO) agonist (SAG), which binds to SMO and activates the $\mathrm{HH}$ pathway, and measured the expression of ACTRT1. GLI1 and PTCH1 mRNA expression was used as controls of HH pathway activation. GLI1 and PTCH1 mRNA responded well upon SAG treatment in proliferating and serum-starved cells (Fig. 2a,b respectively). The expression of ACTRT1 mRNA and ARP-T1 protein was increased by SAG treatment in proliferating (Fig. 2a) and differentiating (Fig. 2b) conditions. We used additional SMO activator, purmorphamine, to confirm the results with SAG treatment, under differentiating condition. In this case, we also used vismodegib (SMO inhibitor used for the treatment of BCC) and both purmorphamine and vismodegib, to understand better the regulation of ARP-T1 by the $\mathrm{HH}$ pathway. ACTRT1, GLI1 and PTCH1 mRNA increased about 2 fold upon purmorphamine treatment similar as the treatment with SAG_Fig. 2c-e). GLI1 and PTCH1 expression decreased upon both purmorphamine and vismodegib treatment as expected (Fig. 2d,e), but not the expression of ACTRT1 indicating that ACTRT1 mRNA expression is regulated by a non-canonical $\mathrm{HH}$ pathway, although ARP-T1 protein expression was similar to the basal level in this condition (Fig. 2c).

A cascade of phosphorylation is known to be involved in $\mathrm{HH}$ signaling pathways, e.g. PKC, GSK$3 \beta$, S6K, PKA, PI3K, aPKC-I/ $\lambda^{8}$. To investigate the HH pathway in which ARP-T1 plays a role in BCC onset, differentiated NHEK, in which ARP-T1 is highly expressed, were treated with different kinase inhibitors. The cells treated with GF109203X known as PKC and PKA inhibitor showed significantly decreased expression of ACTRT1 mRNA, while cells treated with rapamycin (mTOR/S6K inhibitor), LY294002 (PI3K inhibitor) and AG1478 (epidermal growth factor tyrosine kinase inhibitor) showed increased expression of ACTRT1 mRNA (Fig. 2f). We further narrowed down to treat NHEK and HaCaT cells with Gö6976 (PKC alpha/beta inhibitor), Rottlerin (PKC delta inhibitor) and ZIP 2549 (atypical PKC inhibitor). We found that Rottlerin inhibits the expression of ACTRT1 mRNA in both NHEK and HaCaT cells, indicating that ACTRT1 mRNA expression is regulated by PKC delta (Fig. 2g,h). Two different programs (PhosphositePlus and Swiss Institute of Bioinformatics) predicted several putative phosphorylation sites in ARP-T1 WT and the mutant protein (from ACTRT1547_548insA mutation) (Fig. 2i). One of the predicted phosphorylation sites in C-terminus at $\mathrm{S} 352$ position showed similar consensus phosphorylation site specificity with $\mathrm{PKC}$ (www.kinexus.ca). We transduced ACTRT1 (WT), ACTRT1547_548insA (M) and vector control (V) tagged with DDK (same sequence as FLAG) into differentiated NHEK and immunoprecipitated using anti-FLAG M2 affinity gel. We used the Phospho-(Ser) PKC substrate antiserum, which recognizes proteins only when phosphorylated at serine residues surrounded by arginine or lysine at the -2 and +2 positions and a hydrophobic residue at the +1 position (ARP-T1 S352 : MSS*FKQ). This antiserum recognized ARP-T1 WT but not ARP-T1 mutant suggesting that ARPT1 is indeed phosphorylated by PKC and stable while ARP-T1 mutant, which lack the phosphorylation site, is not phosphorylated by PKC (Fig. 2j). 
Park_Fig. 2

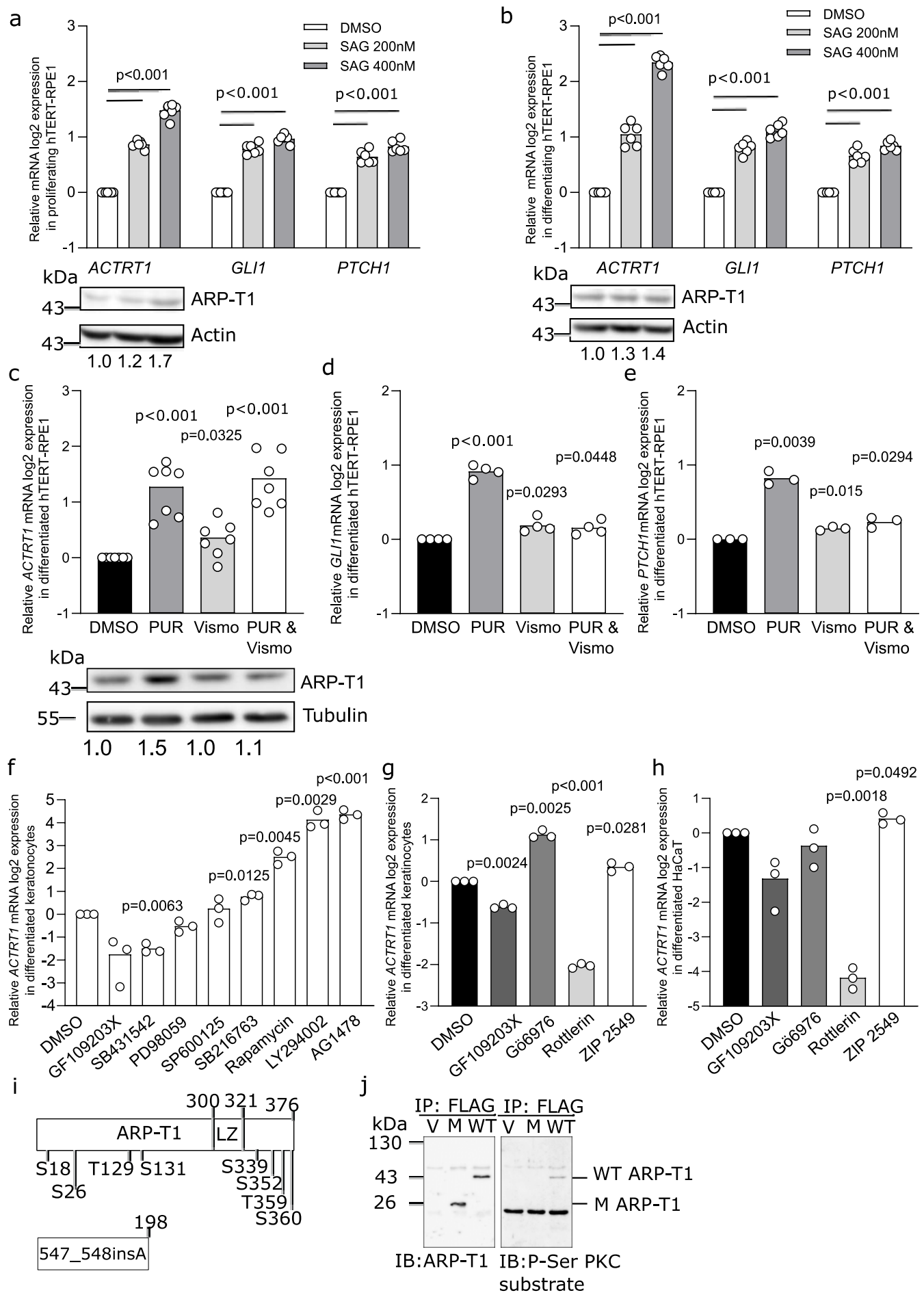




\section{ARP-T1 interacts with proteins involved in ciliogenesis.}

146 To understand the molecular pathways exploited by ARP-T1, we performed coimmunoprecipitation followed by mass spectrometry (MS) analysis to identify proteins interacting with ARP-T1. We transduced ACTRT1 (WT), ACTRT1 547_548insA (M) and vector control (V) tagged with DDK into the cells and immunoprecipitated using anti-FLAG M2 beads. We found putative interacting proteins in HeLa cells (Table 1, Fig. 3a), differentiated hTERT-RPE1 cells (Table 1, Fig. 3b), and NHEK (Table 1). ARP-T1 interacts in all cells with proteins involved in PC structure. We confirmed these interactions by immunoblot analysis with acetylated-tubulin, TCP8 (one of 8 subunits of chaperonin-containing T-complex), HSC70, BAG2, gamma-tubulin, EHD4, septin 2 and septin 9 antisera (Fig. 3a,b). While chaperones are often identified to some extent in MS analysis of precipitates with overexpressed proteins, the high number of spectral hits (Table 1) and the location of septins and EHD4 at ciliary basal body led us to observe the localization of ARP-T1 in ciliated hTERT-RPE1 cells.

We analyzed ciliogenesis, which was induced by serum starvation and differentiation, in ARPE19 and hTERT-RPE1 cells. ARP-T1 localizes to the ciliary base when we co-stained ARP-T1 with acetylated-tubulin, which stains ciliary axoneme. ARP-T1 co-localized with rootletin, a major basal body protein, in PC (Fig. 3c and Extended data Fig. 1d in HaCaT cells). ARP-T1 interacts and forms a complex with gamma-tubulin, which localizes in the vicinity of the basal body (Fig. $3 \mathrm{~d}$ and Extended data Fig. 1e in HaCaT cells). We also confirmed that ARP-T1 co-localizes with EHD4 and septin 2 (Fig. 3d). We used proximity ligation assays to further confirm these interactions (Extended data Fig. 1f and 1g). To reinforce these results, we chose to overexpress EHD4 and then locate ARP-T1. We transfected hTERT-RPE1 cells with EHD4 (EHD4) and vector control (V) tagged with V5. After immunoprecipitation using anti-V5 beads and immunoblot, we detected ARP-T1 in the EHD4 samples but not in the vector control (Extended data Fig. 1h). In the same cells, we confirmed that ARP-T1 co-localizes with EHD4 (Extended data Fig. 1i). 
a

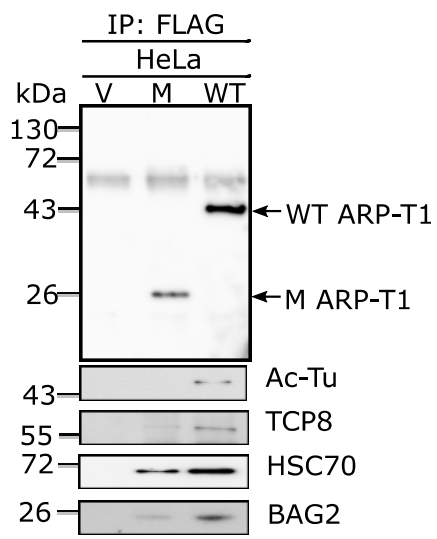

b

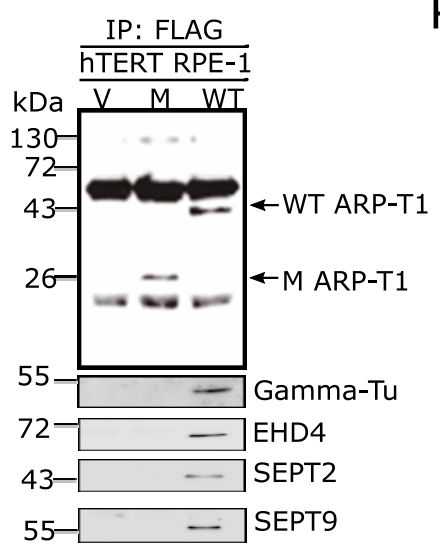

\section{Park_Fig 3}
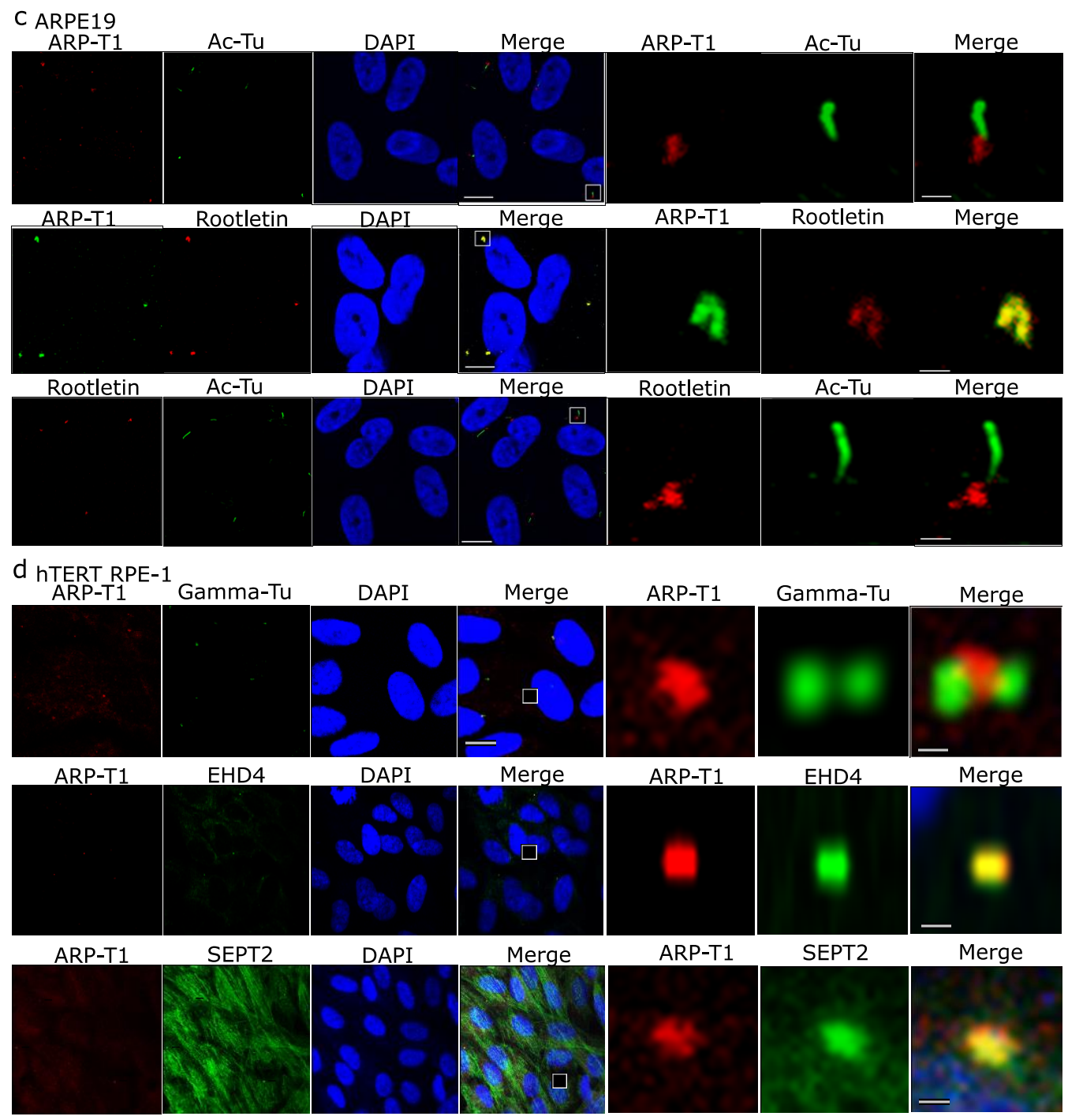


\begin{tabular}{|c|c|c|c|}
\hline HeLa cells & ARP-T1 & 547_548 InsA & Vector \\
\hline ARP-T1 & 183 & 91 & 0 \\
\hline Heat shock cognate $71 \mathrm{kDa}$ protein & 121 & 68 & 9 \\
\hline T-complex protein 1 subunit theta (TCP1 theta) & 66 & 20 & 0 \\
\hline RuvB-like 2 & 73 & 39 & 1 \\
\hline Erlin-2 & 28 & 16 & 0 \\
\hline BAG family molecular chaperone regulator 2 (BAG 2) & 91 & 33 & 4 \\
\hline Ras-related protein Rab-8A & 7 & 4 & 0 \\
\hline Dynactin & 19 & 3 & 0 \\
\hline Importin subunit $\alpha-1$ & 18 & 7 & 0 \\
\hline Exportin 1 & 19 & 2 & 0 \\
\hline Filamin-A & 11 & 5 & 0 \\
\hline \multicolumn{4}{|l|}{ NHEK } \\
\hline ARP-T1 & 139 & 104 & $\underline{0}$ \\
\hline Heat shock $70 \mathrm{kDa}$ protein & $\underline{38}$ & 19 & $\underline{\underline{0}}$ \\
\hline BAG family molecular chaperone regulator 6 (BAG 6) & $\overline{13}$ & $\overline{6}$ & $\underline{0}$ \\
\hline T-complex protein 1 subunit beta (TCP1 beta) & $\overline{9}$ & $\underline{\underline{0}}$ & $\underline{\underline{0}}$ \\
\hline Erlin-2 & $\underline{8}$ & $\underline{\underline{3}}$ & $\underline{0}$ \\
\hline Tubulin-6 & $\overline{7}$ & 3 & $\underline{0}$ \\
\hline Rab18 & $\overline{7}$ & $\underline{\underline{2}}$ & $\underline{\underline{0}}$ \\
\hline BAG 2 & $\underline{5}$ & 1 & $\underline{0}$ \\
\hline \multicolumn{4}{|l|}{ hTERT-RPE } \\
\hline BAG2 & 45 & 14 & 1 \\
\hline TCP1 theta & 19 & 0 & 0 \\
\hline RuvB-like 1 & 14 & 4 & 0 \\
\hline EH domain containing protein 4 (EHD4) & 16 & 1 & 0 \\
\hline Septin-2 & 12 & 2 & 1 \\
\hline Septin-9 & 6 & 1 & 0 \\
\hline Rab10 & 10 & 3 & 1 \\
\hline PDZ and LIM domain protein 5 (PDLI5) & 10 & 0 & 0 \\
\hline Rab6A & 5 & 0 & 0 \\
\hline EH domain containing protein 1 (EHD1) & 5 & 0 & 0 \\
\hline Actin related protein 2 & 4 & 1 & 0 \\
\hline Cortactin & 11 & 9 & 4 \\
\hline
\end{tabular}

176

177

Table 1

178

179 
ARP-T1 associates with a ciliopathy in BDCS.

Based on the localization of ARP-T1 to the basal body and the implication of septin 2 in ciliary length control ${ }^{24}$, we analyzed the ciliary structures in the samples from BDCS patients (ACTRT1 547_548insA, mutations B2, mutation A3, mutation CNE12) ${ }^{6}$ comparing to normal hair follicles and samples from a sporadic BCC patient. First, we observed the ciliary structures by staining with rootletin and acetylated-tubulin (Fig. 4a, top) and confirmed the co-localization of ARP-T1 and rootletin (Fig. 4a bottom, individual stainings with rootletin, acetylated-tubulin, or ARP-T1 are shown in Extended data Fig. 2 and 3). Strikingly, the ciliary length (Fig. 4b) and fluorescence intensity of rootletin and ARP-T1 (Fig. 4c,d) were significantly reduced in the BDCS samples compared to normal hair follicles and sporadic BCC. Ciliary length, rootletin fluorescence and ARP-T1 fluorescence were particularly reduced in ACTRT1 547_548insA (insA in Fig. 4b-f) and B2 mutation while less prominently reduced in mutations $A 2$ and CNE12. We also found significant correlations between ARP-T1 fluorescence and ciliary length (Fig. 4e) and between ARP-T1 fluorescence and rootletin fluorescence (Fig. 4f). We concluded that BDCS, inherited BCC, unlike sporadic BCC, is a novel ciliopathy and the role of ARP-T1 as a part of cilia is to prevent the disease.

To study the role of ARP-T1 in controlling ciliary length in vitro, we overexpressed ACTRT1 WT and ACTRT1 547 548insA in hTERT-RPE1 cells. The length of PC was quantified based on acetylated-tubulin staining. ARP-T1 mutant overexpression reduced the ciliary length, approximately by $25 \%$ (Fig. $4 \mathrm{~g}$ and quantified in Fig. $4 \mathrm{~h}$, individual stainings are in Extended data Fig. 4a). We then investigated more closely the role of ARP-T1 with ACTRT1 silencing in hTERTRPE1 cells. Two different ACTRT1 shRNAs efficiently knocked down the expression of ACTRT1 at mRNA level (65\% for KD1 and $50 \%$ for KD2; shown in Extended data Fig. 4c) and at ARP-T1 level (30\%, Fig. 4k and Extended data Fig. 4d). PC in ACTRT1-depleted cells were shorter than in control cells ( $25 \%$ reduction; Fig. $1 \mathrm{i}, \mathrm{j})$. These results were confirmed in $\mathrm{HaCaT}$ cells, in which ciliary length was halved with ACTRT1 KD1 and KD3 (Extended data Fig. 5a-c). To further confirm the role of ARP-T1 mutant in the control of ciliary length, we expressed ACTRT1 547 548insA resistant shRNA (MshR) in control and ACTRT1 KD cells. ARP-T1 MshR expression leads to an identical reduction of ciliary length in control and KD cells (shown by the quantification in Fig. 4I, stainings are shown in Extended data Fig. 6a). These results confirmed that ARP-T1 loss of function and ACTRT1 silencing both lead to the reduction of the ciliary length. Therefore, ARP-T1 is required to maintain the normal length of cilia in vitro. In summary, ARP-T1 is located in ciliary basal body and regulates ciliogenesis both in vivo and in vitro. Absence of ARP-T1 gives rise to an epidermal ciliopathy. 


\section{Park_Fig 4}

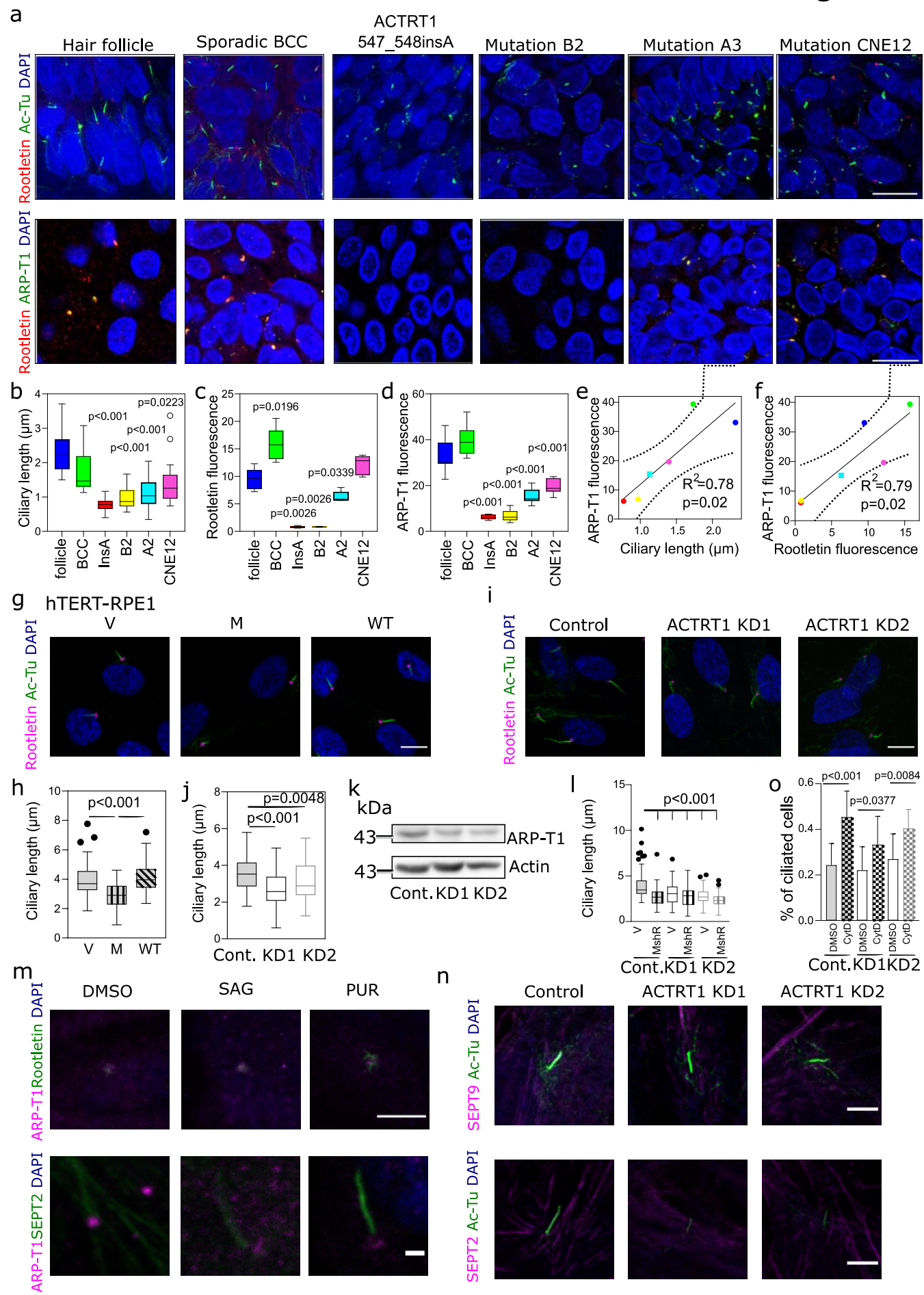


ARP-T1 favors for septin 2 localization in the axoneme, without affecting the actin cellular network.

$\underline{P C}$ is essential for $\mathrm{HH}$ pathway, and we described that ARP-T1 is localized at its basis and is necessary for the full length of the axoneme. Thus, we investigated the localization of ARP-T1 and its interacting proteins during $\mathrm{HH}$ activation. We found that ARP-T1 remains at the cilia basal body upon SMO activation, with SAG and purmorphamine, according to the co-localizations with rootletin and septin 2 (Fig. $4 \mathrm{~m}$ ). We also showed that remaining ARP-T1, in the ACTRT1 KD cells, still co-localizes with the rootletin, and septins 2 and 9 (Extended data Fig. 6b) and that septin 9 localized to the axoneme, septin filaments and the base of the PC in control and ACTRT1 KD cells under differentiation (Fig. $4 \mathrm{n}$ top). Therefore, ARP-T1 decrease does not affect neither its interactions with rootletin and septins nor septin 9 localization. Unexpectedly, we observed that septin 2, normally localized in the axoneme under serum-starvation, is no longer there in ACTRT1 KD cells (Fig. $4 \mathrm{n}$ bottom). We were able to detect septin 2 in its other locations, i.e. septin filaments and the base of the PC.

Finally, as the actin cytoskeleton network is involved in ciliogenesis and as we found interactions between ARP-T1 and several proteins associated with this network (cortactin, septins, ARP2), we studied the actin cytoskeleton in ACTRT1 KD cells and its involvement in cilia formation. We did not find any difference in actin filaments comparing ACTRT1 KD and control cells (unpublished data). Moreover, cytochalasin D-induced ciliogenesis ${ }^{25}$ was not blocked by silencing of ACTRT1 (Fig. 40), indicating that the actin cytoskeletal network is not deficient in these cells and is unlikely to be the cause of ciliary shortening.

\section{ARP-T1 is located in the midbody in dividing cells.}

It was reported that septin 2 acts as a scaffold for myosin II and its kinases at the cleavage furrow ${ }^{26}$ and that septin 9 mediates midbody abscission during cytokinesis ${ }^{27}$. Therefore, we searched for the localization of ARP-T1 in dividing cells. ARP-T1 localized at the midbody in hTERT-RPE1 cells (Fig. 5a), HeLa cells (Fig. 5b) and HaCaT cells (Fig. 5c). To determine whether ARP-T1 was implicated in cell division, we performed proliferation and apoptosis assays. Using EdU, an analog of thymidine, we found that ARP-T1 is not necessary for proliferation because control and ACTRT1 KD cells incorporated identical amounts of EdU (Fig. $5 \mathrm{~d}$ ). We then analyzed apoptosis of these cells. ARP-T1 also has no effect on the different stages of apoptosis, although we observed a slight decrease in the live cell population with KD2 (Fig. 5e). In our conditions, ACTRT1 silencing has no obvious impact on cell division. Postmitotic midbody is directly implicated in primary ciliogenesis. After cytokinesis, the midbody remnant moves along the apical surface, proximal to the centrosome at the center of the apical surface where the PC emerges. The midbody remnant carries RAB8, IFT20, IFT88 and exocyst subunits required for ciliogenesis. If the remnant is removed, primary ciliogenesis is greatly impaired ${ }^{28}$. Midbody remnant licenses PC formation ${ }^{29}$. Both midbodies and PC contain acetylated tubulin, many proteins in the midbody can also be found at the base of the cilium in the centrioles ${ }^{30}$ (Fig. $\left.5 f-i\right)$. 


\section{Park_Fig 5}

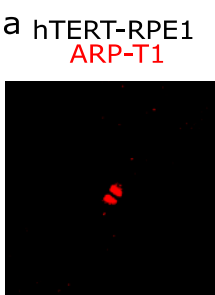

C HaCaT ARP-T1

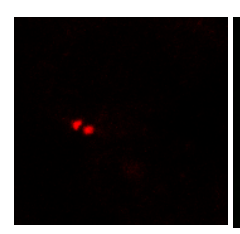

f
Ac-Tu

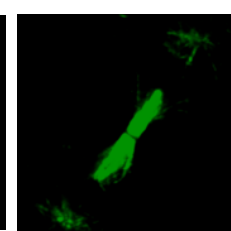

Ac-Tu

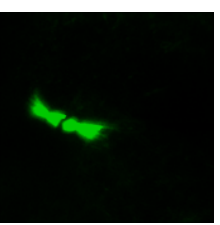

g
Merge with DAPI

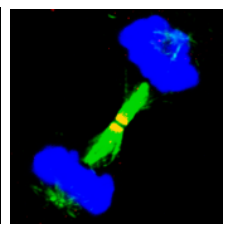

Merge with DAPI

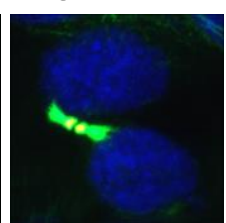

b HeLa

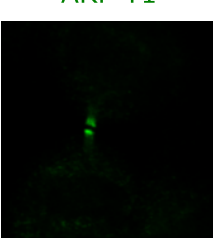

$d_{\text {hTERT-RPE1 }}$

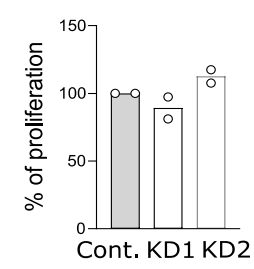

Ac-Tu
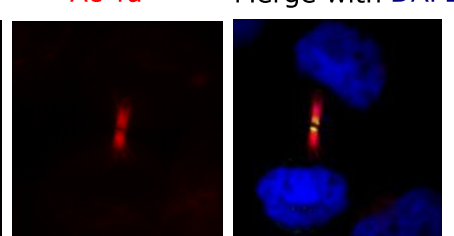

e hTERT-RPE1

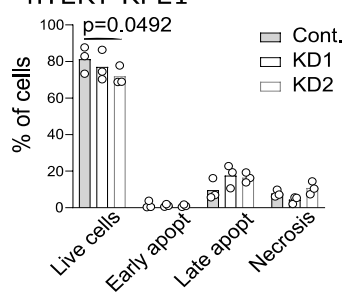

h

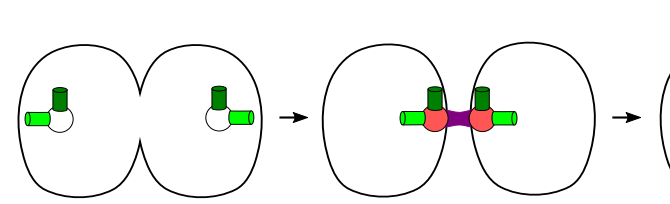

i

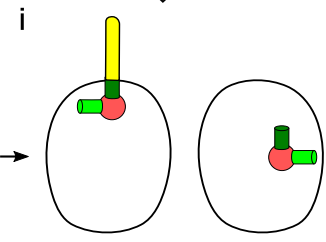

Mother centriole $\square$ Daughter centriole $\bigcirc$ ARP-T1 Primary cilia

\section{DISCUSSION}

Here, we report that ARP-T1 is a ciliogenesis protein located at the basal body of PC. BDCS tumors and ACTRT1-deficient cells show shortened PC. Thus, BDCS is a novel and first ciliopathy implicated in skin cancer caused by mutations of ACTRT1 or its enhancer RNA elements. ARPT1 supports intact cilia and controls proper ciliogenesis, potentially through septin 2 involvement. ARP-T1 is highly expressed during epidermal differentiation, is stabilized by protein kinase $\mathrm{C}$ and ACTRT1 expression is regulated by PKC delta and by a non-canonical $\mathrm{HH}$ pathway.

ACTRT1/ARP-T1 regulation has not been described yet. Here, we show that $\mathrm{HH}$ and mTORC2/PKC delta pathways positively regulate ACTRT1 at the mRNA level. HH signaling is activated upon cell differentiation, at the cilium, and is known to induce canonical and noncanonical signaling pathways. As ARP-T1 was previously reported to activate the $\mathrm{HH}$ signaling ${ }^{6}$, it was important to decipher a possible feedback loop with this pathway. We found that SMO activation favors ACTRT1 mRNA and ARP-T1 protein expression. However, a blockade with vismodegib, used in the clinic to treat $\mathrm{BCC}$ patients, could not reverse purmorphamine-induced ACTRT1 mRNA expression. We concluded that ACTRT1 mRNA is regulated by a non-canonical $\mathrm{HH}$ signaling. We next unraveled which phosphorylation pathway was implicated in this noncanonical HH signaling. MAP kinase inhibition with PD98059 had no effect on ACTRT1 mRNA expression. By using PI3K/mTORC1/S6K and mTORC2/PKC delta inhibitors, we found that the proliferating signaling $\mathrm{PI} / \mathrm{K} / \mathrm{mTORC} 1 / \mathrm{S} 6 \mathrm{~K}$ limits ACTRT1 mRNA expression while its differentiating counterpart mTORC2/PKC delta ${ }^{28}$ favors it. These findings highlight the role of $\mathrm{HH}$ and mTORC2/PKC delta pathways in ACTRT1/ARP-T1 regulation. 
By MS analysis, we identified a machinery of molecular ARP-T1 interactors involved in PC formation (see Table 1): (1.) Chaperon containing TCP-1 (CCT/TRiC), consists of two identical stacked rings, each containing eight subunits (TCP1-8). This complex folds various proteins including actin and tubulin. It was shown that CCT/TRiC family chaperonin localizes to centrosomes, forms a complex with BBS proteins BBS6, BBS10 and BBS12, and mediates the assembly of the BBSome, involved in ciliogenesis regulating transports of vesicles to the cilia ${ }^{29}$. This BBSome protein complex binds to Rabin 8, the GTP/GDP exchange factor, for the small GTPase RAB8. RAB8 localizes to the cilium, is required for ciliogenesis, and mediates the docking and fusion of vesicles. It was proposed that the BBSome acts upstream of RAB8 in this vesicular transport to the cilium ${ }^{30}$. BBS causing IFT27 mutations point to a link of the BBSome with IFT where this small GTPase might be involved in delivering cargo from the BBSome to the IFT ${ }^{31}$ (2.) CCT and HSC70 form a stable complex and this interaction was suggested to be used to deliver the unfolded substrate from HSC70 to the substrate-binding region of CCT ${ }^{32}$. HSC70 interacts with IFT88 ${ }^{33}$. Bcl2-associated athanogen (BAG2) protein forms a chaperone complex with HSC70 and regulates their protein folding activity ${ }^{34}$; (3.) The Eps15 homology domain (EHD) family of proteins, composed of EHD1-4, is associated with RAB8 and RAB11 membranes and regulates endosomal membrane trafficking ${ }^{35}$. EHD4 locates to the transition zone of cilia and plays a role in protein transport to cilia and ciliogenesis. Ehd4 deficient mice show a strong phenotype in skin, kidney and testis with small testis, reduced sperm motility, sperm number and germ cells, such that Ehd4-/- mice are subfertile ${ }^{36}$. Ehd4 deficient mice suffer from hypotrichosis at the dorsal back and have a PC phenotype (Dr. Markus Plomann, unpublished data); (4.) Septin 2 localizes to the base of the axoneme. Cells completely depleted of septin 2 lack a cilium whereas cells partially depleted at the base of PC have a significantly shortened cilium than control ${ }^{24}$. Septin 2 interacts with $\mathrm{HSC} 70^{37}$. Septin 9 has been shown to interact with microtubules ${ }^{38}$ and localizes to the ciliary pocket region ${ }^{39}$. PC in septin 9 depleted cells are shorter than that in control cells ${ }^{39}$. Gammatubulin associates with centrosomes and localizes to the vicinity of basal body ${ }^{40}$.

Overall, our MS analysis results point to a function of ARP-T1 in the ciliary basal body at the crossroads of vesicle trafficking, protein folding and the cytoskeleton (Supplementary Table 1). The ciliary pocket or periciliary PM serves as an interface with the actin cytoskeleton, which is important for the movement of the basal body to the PM and for vesicular and endosomal trafficking $10,41,42,43$. We hypothesize that ARP-T1 is functional at this interface and may stabilize the cytoskeletal matrix.

Functional genomic screening showed that proteins involved in actin dynamics and endocytosis are important for ciliogenesis. Depletion of gelsolin family proteins (GSN and AVIL), which regulate cytoskeletal actin organization by severing actin filaments, reduced the number of ciliated cells and silencing of ACTR3 (ARP-3), a major constituent of the ARP2/3 complex involved in nucleating actin polymerization, increased cilium length ${ }^{44}$. On the other hand, it was reported that the actin cytoskeleton is disrupted in KD cell lines targeting ciliary proteins. For example, KD cells of Ahi1, whose human ortholog is mutated in Joubert syndrome, showed disorganizing and decrease in actin filaments ${ }^{45}$. ARP-T1 has $49 \%$ identity with $\beta$-actin and $40 \%, 37 \%$, 29\% identity with ARP1, ARP-2, ARP-3, respectively. ARP-T1 as a basal body protein is involved in a positive regulation of ciliogenesis. Similar to ARP-T1, some cancer-associated mutations result in ciliary resorption: (1.) Von Hippel-Lindau (VHL) disease is characterized by the development of premalignant renal cysts, and arises because of functional inactivation of $\mathrm{VHL}$ tumor suppressor protein ( $\mathrm{pVHL}$ ). pVHL maintains the structural integrity of the PC for suppression of uncontrolled proliferation of kidney epithelial cells and cyst formation ${ }^{46}$. (2.) It was shown by electron microscopy that glioblastoma cells and tumors have immature PC and basal body/centrioles ${ }^{47,48}$. (3.) Mutations in 
333 Budding uninhibited by benzimidazole-related 1 (BUBR1), a molecule of spindle assembly checkpoint, cause premature chromatid separation (mosaic variegated aneuploidy), cancer disposition and impaired ciliogenesis ${ }^{49}$. Despite all these clues, we could not detect a difference in the actin filaments between ACTRT1 KD and control cells, and our cytochalasin D- induced ciliogenesis experiments did not allow us to confirm a role of the actin cytoskeletal network in the shortened cilia observed in silenced ACTRT1 cells.

Diffusion barrier is another cellular structure essential for the integrity of the PC ${ }^{38}$. Septins are key components of the diffusion barrier at the base of the PC, forming ring-like structures and being transported to the axoneme. The involvement of septin 2 in ciliogenesis was particularly studied these last years $24,39,50$. In an attempt to understand how the knockdown of ACTRT1 led to a shortening of ciliary length, we observed that septin 2 was absent from the axoneme in ACTRT1 silenced cells, but preserved in septin filaments and in the base of the PC. Such a situation was different for septin 9, whose location remained unchanged, i.e. in the axoneme, septin filaments and the base of the PC. These results suggest that the ciliogenesis defect observed in ACTRT1 deficient cells could be due to a default in the diffusion barrier mediated by septin 2. We are currently investigating the link between septin 2 and ARP-T1 in cilia formation, notably using SMO in/out assay ${ }^{51}$ and FRAP experiments ${ }^{39}$. These results will be part of a forthcoming publication.

Septins are not only essential for the diffusion barrier at the base of PC, but also participate in cell division, where they are located in the midbody ${ }^{27}$. Our investigations have shown that ARP-T1 is also found in the midbody of dividing cells, and that it could therefore have an impact on cell division. Nevertheless, cell proliferation and apoptosis were not affected by the decrease of ARPT1, and thus ARP-T1 at the midbody level has no effect on cell division. This was not unexpected, as Pejskova et al. ${ }^{52}$ recently described that KIF14, a protein involved in ciliogenesis, was also localized in the midbody without any impact on cell division.

With our results, we propose a model where ARP-T1 leads primary ciliogenesis via direct interaction with proteins in ciliary machinery. First, ARP-T1 is localized in the mitotic spindle through the interaction with gamma-tubulin, which is present in between the mother centriole and the daughter centriole (Fig. $\underline{5 f}$ ). As the cytokinesis proceeds, the complex of ARP-T1 and two centrioles moves to the central spindle where the midbody is formed by recruiting septins (Fig. $\underline{5 \mathrm{~g}}$ ). The midbody cleaves one side of the intracellular bridge and remains tethered to one of the daughter cells. The complex of ARP-T1, two centrioles and midbody move towards the apical

364 surface of daughter cells where PC will be formed (Fig. $\underline{5 h, i) .}$

365 Dynamic $\mathrm{HH}$ signaling has been identified in mammalian post-natal testis, and $\mathrm{HH}$ pathway components PTCH1, SUFU and GLI1 were identified in spermatogonia, spermatocytes and spermatids ${ }^{53}$, where spermatids express high levels of SUFU to repress $\mathrm{HH}$ activity ${ }^{54}$. It was previously reported that ARP-T1 binds to the GLI1 promoter and inhibits GL/1 mRNA expression signaling ${ }^{6}$. In the current study, we showed that the elevated level of ACTRT1 mRNA with purmorphamine was not decreased by the treatment with purmorphamine and vismodegib (Fig. $2 \mathrm{c}-\mathrm{e})$. Nevertheless, this regulation is absent at the ARP-T1 protein level, as purmorphamineinduced ARP-T1 expression was counteracted by the addition of vismodegib (Fig. 2c). Thus, we hypothesize that ACTRT1 functions in a SMO-independent $\mathrm{HH}$ pathway and that posttranscriptional modifications, as shown for pKC, regulate ARP-T1 function. The activation of a noncanonical $\mathrm{HH}$ pathway may explain the apparent paradox that we observed shortened $\mathrm{PC}$ both in $\mathrm{BCC}$ from BDCS and in ACTRT1 KD cells. While loss of PC is observed in diverse cancers where it correlates with worse prognosis, their retention is paramount for medulloblastoma and BCC 
BCC mouse models ${ }^{13}$, and was recently linked to development of resistance to SMO-inhibition ${ }^{55}$. This suggest that BDCS is a model for the study of vismodegib resistance in BCC and medulloblastoma.

Functions of the PC, including activation of the $\mathrm{HH}$ signaling, are dependent on the ciliary length and on proteins involved in ciliogenesis, such as proteins located to the basal body ${ }^{56}$. In addition to this, ARP-T1 was reported to regulate the $\mathrm{HH}$ pathway ${ }^{6}$. Here, as we demonstrated that ACTRT1 silencing is associated with a shortened $\mathrm{PC}$, we assessed the expression of $\mathrm{HH}$ proteins in our deficient cells. GLI1 and PTCH1 mRNA expression tends to increase but not significantly in ACTRT1 KD cells (Extended data Fig. 6c,d), suggesting that our silencing might not be sufficient to correctly activate the $\mathrm{HH}$ signaling, or that another hit might be needed for a proper activation. In order to look for a second hit necessary for a strong activation of GL/1, we studied in depth the transcriptomic analysis performed in Bal et al. ${ }^{6}$, and we unraveled a higher expression of ARHGAP36 mRNA in BDCS samples, with an approximately 17-fold increase. ARHGAP36 gene is located to the $\mathrm{X}$ chromosome, in the close proximity of ACTRT1 gene, and is known to activate the $\mathrm{HH}$ pathway in medulloblastoma ${ }^{57}$. ARHGAP36 might contribute to GL/1 activation in BDCS patients. Another gene of interest is SMARCA1. Indeed, SMARCA1 expression is often lost in gastric cancer cells due to methylation ${ }^{58}$ and in soft tissue sarcomas ${ }^{59}$. This gene is located next to ACTRT1 gene, and it cannot be excluded that mutations in the RNA elements regulating ACTRT1 do not also affect the expression of SMARCA1, which expression is 1.8-fold higher in BDCS patients ${ }^{6}$. SMARCA1 encodes for the probable global transcription activator SNF2L1, which act as a chromatin remodeler and interacts with actin. Loss of expression of SMARCA1 increased proliferation of gastric cancer cells, giving it a tumor suppressor role ${ }^{58}$. A whole exome sequencing of BDCS tumors is presently on going and we are currently studying the link between ARHGAP36, SNF2L1, and ARP-T1. These results will be the focus of a future publication.

Our results show that ARP-T1 acts as a direct or indirect actor in a non-canonical HH pathway and bridges between actin cytoskeleton organization involved in vesicle transport at the centrosome, the centrioles and the basal body, and the formation of PC to prevent the pathogenesis of BDCS. Above all, our studies shed light on how ciliogenesis is controlled in carcinogenesis by ARP-T1. ARP-T1 could be a new target for novel therapeutic approaches in BDCS and BCC, the most frequent human cancer. This appears particularly important since loss of $P C$ is a recognized mechanism of resistance to SMO inhibitors in medulloblastoma and BCC ${ }^{55,60}$. Finally, Glaessl et al. ${ }^{61}$ described BDCS BCCs to be more aggressive and prone to relapse. As BDCS patients have a shortened PC, we may hypothesize that BDCS BCCs are a more advanced form of BCC. 


\section{Online METHODS}

\section{Cell Culture and samples}

418 Normal human epidermal keratinocytes (NHEK, established from neonatal foreskin in our 419 laboratory) and immortalized keratinocytes, HaCaT cells (Invitrogen, Basel, Switzerland), were 420 grown in EpiLife medium (Gibco, Invitrogen) with Human Keratinocyte Growth Supplement (Gibco, 421 Invitrogen), $10 \mu \mathrm{g} / \mathrm{ml}$ gentamicin and $0.25 \mu \mathrm{g} / \mathrm{ml}$ amphotericin B (Gentamicin / Amphotericin B 422 solution, Gibco, Invitrogen). Spontaneously immortalized adult retinal epithelial cell line 19 (ARPE423 19) and immortalized hTERT-RPE1 cells (ATCC, Manassas, VA) were cultured in Dulbecco's 424 Modified Eagle's Medium (DMEM) High Glucose, GlutaMAX TM, Pyruvate (Gibco, Invitrogen) or DMEM/F12 (ATCC) with $0.01 \mathrm{mg} / \mathrm{ml}$ hygromycin B (Sigma-Aldrich, St Louis, MO), both media supplemented with $10 \%$ fetal bovine serum (FBS), $100 \mathrm{U} / \mathrm{ml}$ penicillin and $100 \mu \mathrm{g} / \mathrm{ml}$ streptomycin (BioConcept AG, Allschwil, Switzerland). HeLa and HEK293T (Invitrogen) were cultured in DMEM supplemented with $10 \% \mathrm{FBS}, 100 \mathrm{U} / \mathrm{ml}$ penicillin and $100 \mu \mathrm{g} / \mathrm{ml}$ streptomycin. The cells were incubated at $37^{\circ} \mathrm{C}$ in a $5 \% \mathrm{CO}_{2}$ atmosphere.

430 To induce cilia formation, NHEK and HaCaT were cultured up to 7 days in EpiLife media 431 supplemented with $2 \mathrm{mM} \mathrm{CaCl}_{2}$. hTERT-RPE1 and ARPE-19 cells were cultured $48 \mathrm{~h}$ or 35 days 432 respectively in media containing $0.2 \%$ FBS.

433 Bazex-Dupré-Christol syndrome samples were collected and prepared as previously described ${ }^{6}$.

\section{Lentivirus production and transduction}

Lentivirus particles ( $L V s$ ) for $A C T R T 1$ shRNA were produced by Sigma-Aldrich, in pLKO backbone vector. ShRNA sequences are for ACTRT1 KD1: 5' CCG GGC CTG GTT TCT ACC TGT CTA ACT CGA GTT AGA CAG GTA GAA ACC AGG CTT TTT G 3', and ACTRT1 KD2: 5' CCG GGT GCC TTT AGC AAG ACT TAA TCT CGA GAT TAA GTC TTG CTA AAG GCA CTT TTT TG 3'; ACTRT1 KD3: CCG GCA TGA CCT CTA TGA GCA GTT TCT CGA GAA ACT GCT CAT AGA GGT CAT GTT TTT G. Control is an empty vector with same selection.

LVs for ACTRT1 overexpression were produced with calcium phosphate transfection method: ACTRT1 wild-type (WT) and mutant containing the 547-548InsA mutation constructs were produced as previously described ${ }^{6}$. HEK293T cells were transiently co-transfected with psPAX2 (Addgene, Cambridge, MA) and pMD2.G (Addgene) and empty vector or ACTRT1 WT or mutant to produce LVs. LVs were harvested $48 \mathrm{~h}$ later and the titer was determined in HeLa cells ${ }^{62}$. ACTRT1 547-548InsA construct was mutated using QuikChange Multi Site-Directed Mutagenesis Kit (200514, Agilent Technologies, Basel, Switzerland) in the KD1 target (T599G, C602T, C605T) and reverse KD2 target (A341G, A344C, T556G) with following primers (Microsynth, Balgach, Switzerland): KD1 target GCCTGGGTTTTATCTGTCTAA, $\quad$ KD2 $\quad$ target GATTCAGTCTGGCCAAAGGCAC.

HeLa, hTERT-RPE1 and HaCaT cells were transduced using polybrene $(8 \mathrm{ug} / \mathrm{mL}$, TR1003, Millipore), with $\mathrm{LVs} \mathrm{O} / \mathrm{N}$ at $37^{\circ} \mathrm{C}$. The next day, LVs were removed and cells grew in their medium. When necessary, selection with puromycin $(2.5 \mathrm{ug} / \mathrm{mL}, 540411$, Calbiochem) or blasticidin $(7.5$ $\mathrm{ug} / \mathrm{mL}, 15205$, Sigma-Aldrich) started $24 \mathrm{~h}$ later. 


\section{Drug Treatments}

458

459

460

461

462

463

464

465

466

467

468

469

470

471

472

473

474

475

476

477

478

479

480

481

482

483

484

485

486

487

488

489

490

491

492

493

494

495

496

497

498

499

Cells were treated $24 \mathrm{~h}$ with SMO agonists 200 and 400 nM SAG (566660, Calbiochem) and 3 $\mu \mathrm{M}$ purmorphamine (4551, Tocris bioscience), or antagonist $5 \mu \mathrm{M}$ vismodegib (S1082, Selleckchem), or kinase inhibitors: $10 \mu \mathrm{M}$ GF109203X (ALX-270-049, Enzo Life Sciences), $10 \mu \mathrm{M}$ SB431542 (1614, Tocris bioscience), $20 \mu \mathrm{M}$ PD98059 (9900, Cell Signaling Technologies), $5 \mu \mathrm{M}$ SP600125 (270-339-M005, Alexis Biochemicals), $10 \mu \mathrm{M}$ SB216763 (1616, Tocris bioscience), 200 nM Rapamycin (R0161, LKT Laboratories), 50 MM LY294002 (70920, Cayman Chemical), 5 M AG1478 (270-036-M001, Alexis Biochemicals), 3 MM Gö6976 (12060S, Cell Signaling Technologies), $10 \mu \mathrm{M}$ Rottlerin (1610, Tocris bioscience), $20 \mu \mathrm{M}$ ZIP (2549, Tocris bioscience, kind gift from Prof. Anthony Oro, Stanford University School of Medicine).

To induce cilia in proliferating condition, hTERT-RPE1 cells were treated for $16 \mathrm{~h}$ with $50 \mathrm{nM}$ cytochalasin D (250255, MERCK).

\section{Western blot}

NHEK, HaCaT, ARPE-19, and hTERT-RPE1 cells were harvested in ice-cold FLAG (50 mM Tris/ $\mathrm{HCl} \mathrm{pH} \mathrm{7.5,} 150 \mathrm{mM} \mathrm{NaCl}, 1 \mathrm{mM}$ EDTA, 1\% Triton X-100, and protease inhibitor cocktail [Complete MiniTM tablette, Roche Diagnostics]) or RIPA (50 mM Tris/HCl pH 7.4, $150 \mathrm{mM} \mathrm{NaCl}$, $12 \mathrm{mM}$ Na deoxycholate, 1\% NP-40, 0.1\% SDS, protease inhibitor cocktail, and phosphatase inhibitor cocktail [PhosSTOP, PHOSS-RO, Roche]) lysis buffers for $30 \mathrm{~min}$ on ice with extensively pipetting every $10 \mathrm{~min}$, then spun for 10 to $20 \mathrm{~min}$ at $12,000 \times g$ at $4^{\circ} \mathrm{C}$. Supernatants were collected, heated for $5 \mathrm{~min}$ at $85^{\circ} \mathrm{C}$ and centrifuged. Total protein concentration was determined by BCA assay (PierceTM BCA Protein Assay Kit; Thermo Scientific, Waltham, MA, USA). Fifteen ug of proteins were loaded onto a 10\% SDS-PAGE gel and electroblotted onto a nitrocelulose or PVDF membrane (Hybond ECL; Amersham, UK). Membranes were incubated overnight at $4^{\circ} \mathrm{C}$ with the following antibodies: anti-ARP-T1 (1:1000, SAB1408334, Sigma-Aldrich / 1:2000, GPSH6, Progen), anti-keratin 10 (1:200, MS-611-P0, Thermo Scientific), anti-IFT88 (1:1000, 139671-AP, Proteintech, UK), anti-actin (loading control, 1:5000, A2066, Sigma-Aldrich) and anti-alphatubulin (loading control, 1:5000, T9026, Sigma-Aldrich). After washes in TBS-T, $1 \mathrm{~h}$ incubation at room temperature (RT) with HRP-secondary antibodies (anti-mouse [1:5000, NA931V, GE Healthcare UK Limited], anti-rabbit [1:5000, NA934V, GE Healthcare], anti-guinea pig [1:5000, ab97155, Abcam]), and revelation with chemiluminescence (ECL Prime, Amersham / WesternBright Quantum, Advansta, Witec, Sursee, Switzerland), images were acquired using the Luminescent Image Analyzer LAS-4000 mini (Fujifilm, Tokyo, Japan). Band intensity was analyzed with Image $\mathrm{J}$ software (National Institutes for Health, USA).

\section{Co-immunoprecipitation}

Transduced HeLa, NHEK, and serum-starved hTERT-RPE1 cells were washed with PBS and harvested in the FLAG lysis buffer. Supernatants were incubated with $30 \mu \mathrm{L}$ anti FLAG-beads (ANTI-FLAG ${ }^{\mathrm{TM}} \mathrm{M} 2$ Affinity Gel) overnight at $4^{\circ} \mathrm{C}$ and $1 \mathrm{~h}$ at RT on a rotator to pool-down ARP-T1FLAG proteins. Beads were separated by centrifugation for $3 \mathrm{~min}$ at $5000 \times \mathrm{g}$, washed five times with TBS, and eluted in $23 \mu \mathrm{L}$ TBS and $7 \mu \mathrm{L} 5 \mathrm{x}$ SDS-sample buffer at $85^{\circ} \mathrm{C}$ for $5 \mathrm{~min}$. ARP-T1 precipitation was confirmed using ARP-T1 antiserum (1:2000, GP-SH6), and co-precipitated proteins were analyzed using anti-acetylated-tubulin (1:1000, T6793, Sigma-Aldrich), anti-TCP8 / 
TCP1 theta (1:500, PA5-30403, Thermo Scientific), anti-HSC70 (1:200, PA5-27337, Thermo Scientific), anti-BAG2 (1:100, PA5-30922, Thermo Scientific), anti-gamma-tubulin (1:500, ab11316, Abcam), anti-EDH4 (1:1000, kindly provided by Dr. Plomann, unpublished antibody), anti-septin 2 (1:2000, HPA018481, Sigma-Aldrich), anti-septin 9 (1:2000, HPA042564, SigmaAldrich).

\section{Proteomic analysis/ Mass Spectrometry}

Transduced HeLa, NHEK, and serum-starved hTERT-RPE1 cells were washed with PBS and harvested in FLAG lysis buffer, and analyzed by the Proteomic Analysis Facility of University of Lausanne with the following protocol.

\section{Gel separation and protein digestion}

Protein samples were loaded on a $12 \%$ mini polyacrylamide gel and migrated about $2.5 \mathrm{~cm}$ in nonreducing conditions. After Coomassie staining, gel lanes between 15-300 kDa were excised into 5-6 pieces, and digested with sequencing-grade trypsin (Promega) as described by Shevchenko and colleagues ${ }^{63}$. Extracted tryptic peptides were dried and resuspended in $0.05 \%$ trifluoroacetic acid, $2 \%(\mathrm{v} / \mathrm{v})$ acetonitrile for mass spectrometry analyses.

\section{Mass spectrometry analyses}

Tryptic peptide mixtures were injected on a Dionex RSLC 3000 nanoHPLC system (Dionex, Sunnyvale, CA, USA) interfaced via a nanospray source to a high resolution mass spectrometer based on Orbitrap technology: Orbitrap Fusion Tribrid or QExactive Plus instrument (Thermo Fisher, Bremen, Germany), depending on the experiments considered. Peptides were loaded onto

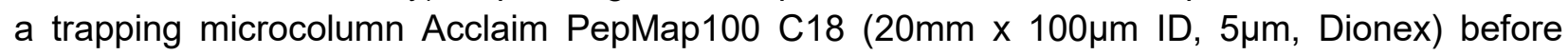
separation on a C18 reversed-phase analytical nanocolumn, using a gradient from 4 to $76 \%$ acetonitrile in $0.1 \%$ formic acid for peptide separation (total time: $65 \mathrm{~min}$ ).

Q-Exactive Plus instrument was interfaced with an Easy Spray C18 PepMap column (25cm x $75 \mu \mathrm{m} I D, 2 \mu \mathrm{m}, 100 \AA ̊$, Dionex). Full MS survey scans were performed at 70,000 resolution. In datadependent acquisition controlled by Xcalibur software (Thermo Fisher), the 10 most intense multiply charged precursor ions detected in the full MS survey scan were selected for higher energy collision-induced dissociation (HCD, normalized collision energy NCE=27\%) and analysis in the orbitrap at $17^{\prime} 500$ resolution. The window for precursor isolation was of $1.5 \mathrm{~m} / \mathrm{z}$ units around the precursor and selected fragments were excluded for 60 s from further analysis.

Orbitrap Fusion Tribrid instrument was interfaced with a reversed-phase C18 Nikkyo column $(75 \mu \mathrm{m}$ ID $\times 15 \mathrm{~cm}, 3.0 \mu \mathrm{m}, 120 \AA$, Nikkyo Technos, Tokyo, Japan) or a custom packed column $(75 \mu \mathrm{m}$ ID $\times 40 \mathrm{~cm}, 1.8 \mu \mathrm{m}$ particles, Reprosil Pur, Dr. Maisch). Full survey scans were performed at a 120 '000 resolution, and a top speed precursor selection strategy was applied to maximize acquisition of peptide tandem MS spectra with a maximum cycle time of $3 \mathrm{~s}$. HCD fragmentation mode was used at a normalized collision energy of $32 \%$, with a precursor isolation window of 1.6 $\mathrm{m} / \mathrm{z}$, and MS/MS spectra were acquired in the ion trap. Peptides selected for MS/MS were excluded from further fragmentation during $60 \mathrm{~s}$.

\section{Data analysis}


MS data were analyzed using Mascot 2.5 (Matrix Science, London, UK) set up to search the Swiss-Prot (www.uniprot.org) database restricted to Homo sapiens taxonomy (UniProt, December 2015 version: 20'194 sequences). Trypsin (cleavage at $K, R$ ) was used as the enzyme definition, allowing 2 missed cleavages. Mascot was searched with a parent ion tolerance of $10 \mathrm{ppm}$ and a fragment ion mass tolerance of $0.50 \mathrm{Da}$ (Orbitrap Fusion) or $0.02 \mathrm{Da}$ (QExactive Plus). Carbamidomethylation of cysteine was specified in Mascot as a fixed modification. N-terminal acetylation of protein and oxidation of methionine were specified as variable modifications.

Scaffold software (version 4.4, Proteome Software Inc., Portland, OR) was used to validate MS/MS based peptide and protein identifications, and to perform dataset alignment. Peptide identifications established at lower than $\mathbf{9 0 . 0 \%}$ probability by the Scaffold Local FDR algorithm were filtered out. Protein identifications were accepted if they could be established at greater than 95.0\% probability and contained at least two identified peptides. Protein probabilities were assigned by the Protein Prophet algorithm ${ }^{64}$. Proteins that contained similar peptides and could not be differentiated based on MS/MS analysis alone were grouped to satisfy the principles of parsimony. Proteins sharing significant peptide evidence were grouped into clusters.

The mass spectrometry proteomics data have been deposited to the ProteomeXchange Consortium ${ }^{65}$ via the PRIDE ${ }^{66}$ partner repository with the dataset identifier PXD016557 and 10.6019/PXD016557.

\section{Immunofluorescence}

Cells grown on coverslips were fixed in $4 \%$ formaldehyde/PBS for $20 \mathrm{~min}$ at RT, then permeabilized in $0.02 \%$ Triton-X/PBS for $10 \mathrm{~min}$. Coverslips were incubated with blocking buffer containing $1 \%$ FBS and $2 \%$ bovine serum albumin $(B S A)$ for $1 \mathrm{~h}$. Primary antibodies (anti-ARP-T1 (1:100, SAB2103464 or SAB1408334; or 1:200, Progen GP-SH6), anti-acetylated-tubulin (1:1000, T6793), anti-rootletin (1:50, sc-67824, Santa-Cruz; 1:200, NBP1-80820, Novus), anti-gammatubulin (1:500, ab11316, Abcam), anti-EDH4 (1:200, kind gift from Dr. Markus Plomann, unpublished antibody), anti-septin 2 (1:200, HPA018481, Sigma-Aldrich), anti-septin 9 (1:200, HPA042564) were diluted in blocking buffer and applied on the cells for $2 \mathrm{~h}$ at RT or overnight at $4^{\circ} \mathrm{C}$. Coverslips were next incubated with secondary antibodies (1:500, A11008, A11001, A21467, A11035, A11003, A11060, Invitrogen; 1:100, BA-9500, Vector Laboratories; 1:200, RPN1233V, GE) for $1 \mathrm{~h}$ at RT. Nucleus was stained with DAPI or Hoechst for $5 \mathrm{~min}$ at RT. Finally, coverslips were mounted onto slides with Dako mounting medium (S3023, Dako Schweitz AG, Baar, Switzerland) and examined with an inverted Zeiss LSM 700 laser scanning confocal microscope equipped with laser diode 405/488/555, SP490/SP555/LP560 emission filters, 2 PMT detectors and Zen2010 software (Zeiss, Feldbach, Switzerland).

Three-dimensional (3D) imaging technique was used to study ciliary imaging. Confocal images were captured at $\sim 0.33-1 \mu \mathrm{m}$ interval using $63 \times / 1.40$ oil objectives. Using Image $\mathrm{J}$ software, the 3D structure was deconvoluted from the Z-stack, and the length, intensity and prevalence of cilia were manually traced and measured. Both cell number and proportion of cells exhibiting PC were determined in approximately five representative fields $\left(100\right.$ to $\left.200 \mu \mathrm{m}^{2}\right)$ for each experimental condition. The mean cilia prevalence was expressed as the percentage of ciliated cells. The average ciliary length was expressed in $\mu \mathrm{m}$. The ciliary rootlet was selected using the freehand tool and the average area was expressed in $\mu \mathrm{m}^{2}$. The average pixel fluorescence intensity of proteins was quantified using the freehand tool to select the area of interest. Twenty to 50 cilia were measured for each experimental condition. 


\section{RNA extraction and Real time quantitative PCR.}

587 Total RNA were harvested using the Qiagen RNeasy Mini Kit (Qiagen, Hombrechtikon,

588

589

590

591

592

593

594

595

596

597

598

599

600

601

\section{Proliferation assay}

603

604

605

606

607

608

609

610

611

612

613

614

615

616

\section{7}

618

619

620

621

622

623

624 $\log 2$ scale.

\section{Apoptosis assay}

Switzerland) according to the manufacturer's instructions. RNA quantity and quality were assessed using the NanoDrop ND-1000 Spectophotometer (Wilmington, USA). RNA were reverse-transcribed using the Primescript RT reagent kit (TakaRa, Saint-Germain-en-Laye, France) in $10 \mu \mathrm{L}$ reaction on a T-Gradient Thermocycler (Biometra, Biolabo, Châtel-St-Denis, Switzerland). Quantitative PCR ( $q P C R)$ was performed on cDNA with Power SYBRGreen PCR Mastermix (Applied Biosystems, CA, USA) on an Applied Biosystems StepOne thermal cycler (Applied Biosystems, Life Technologies, CA, USA). Gene expression was assessed by using ACTRT1 (QT00215642, Qiagen), GLI1 (Fw 5' AGA GGG TGC CAT GAA GCC AC 3', Rev 5' AAG GTC CCT CGT CCA AGC TG 3', Microsynth), PTCH1 (Fw 5' GCT ACT TAC TCA TGC TCG CC 3', Rev 5' TCC GAT CAA TGA GCA CAG GC 3', Microsynth) primers. RPL13A (Fw 5'GCA TCC CAC CGC CCT ACG AC 3', Rev 5' CTC TTT CCT CTT CTC CTC CA 3', Microsynth) was used as endogenous control. Relative gene expression is normalized to the control and reported with a

Proliferation was assessed using the Click-iT EdU Proliferation Assay for Microplates (C10499, Invitrogen). Briefly, cells were plated at $48 \%$ confluency in 96 well black microplate (CLS3603, Corning, MERCK), labelled with $10 \mu \mathrm{M}$ EdU for $24 \mathrm{~h}$, then fixed and clicked according to the manufacturer's instructions. Fluorescence was analyzed with a Mithras LB 940 reader (Berthold technologies, Bad Wildbad, Germany) and MikroWin 2010 software (Berthold technologies) using excitation filter $560 \mathrm{~nm}$ and emission filter $590 \mathrm{~nm}$.

Cells were harvested in Versene (15040, Gibco, ThermoFisher Scientific), then stained with APC Annexin V (550474, BD Pharmingen, San Jose, CA) and Annexin V Apoptosis Detection Kit I (556547, BD Pharmingen) according to the manufacturer's instructions. Results were collected with a FACS Gallios I (Beckman Coulter, Nyon, Switzerland) and analyzed with FlowJo software (BD Life Sciences) at the Flow Cytometry Facility at Unil.

\section{Statistics and Reproducibility.}

We used the one-way ANOVA or the Kruskal-Wallis test, depending on homogeneity of variances, to compare one independent variable in more than two groups. Linear regression was used to compare the correlation between two parameters.

To compare gene expression quantified by RT-qPCR, for each gene, all samples were normalized to the control, and log-2 transformed fold changes comparison to 0 were performed with a onesample t test. To compare protein expression, all samples were normalized to the control, and fold changes comparison to 1 were performed with a one-sample t test. 
625 All experiments were performed at least three times. All statistical analyses were performed using 626 Prism GraphPad (v8, La Jolla, CA, USA).

628 Data Availability

629 Original immunoblots and immunofluorescence data are accessible on Zenodo repository with the 630 dataset identifier 10.5281/zenodo.4317153 (all versions: 10.5281/zenodo.3666278).

\section{SUPPLEMENTARY METHODS}

\section{In situ proximity-mediated ligation assay (PLA)}

634 hTERT-RPE1 cells were seeded on coverslips, fixed and permeabilized. Duolink in situ PLA kit with anti-rabbit PLUS probe and anti-mouse or anti-goat MINUS probe (Sigma-Aldrich) was used according to manufacturer's instructions: blocking for 30 min in a $37^{\circ} \mathrm{C}$ humidified chamber, incubation with primary antibodies (gamma-tubulin (1:500, ab11316), ARP-T1 (1:100, SAB2103464), rootletin (1:50, sc-67824), EHD4 (1:200, ab83859, Abcam), septin 2 (1:100, HPA018481), septin 9 (1:100, HPA042564) for $2 \mathrm{~h}$ at RT or overnight at $4^{\circ} \mathrm{C}$, hybridization with PLA PLUS and MINUS probes (1:5 dilution) for $1 \mathrm{~h}$ at $37^{\circ} \mathrm{C}$, ligation, amplification and final washes. Nucleus was stained with DAPI for 2 min at RT. Coverslips were mounted onto slides and complex formation was examined with an inverted Zeiss LSM 700 microscope.

\section{Transfection of EHD4 plasmid and reverse co-immunoprecipitation}

hTERT-RPE1 cells were transfected using ESCORT IV Transfection Reagent ( $L$ 3287, SigmaAldrich), with pcDNA6.V5 and pcDNA6.V5 hEHD4 WT (kind gift from Dr. Markus Plomann, unpublished construct), according to manufacturer's instructions: first, for $2 \times 10 \mathrm{e} 5$ cells, $1.5 \mathrm{ug}$ DNA and $2 \mathrm{ug}$ ESCORT IV were diluted separately in OPTIMEM (Gibco), then mixed, and incubated 15 min at RT. After two washes in PBS, the mix was drop-wised on the cells, which were then incubated for $6 \mathrm{~h}$ at $37^{\circ} \mathrm{C}$. The transfection was stopped by the addition of $20 \% \mathrm{FBS}$ DMEM/F12 medium and the cells were grown for $48 \mathrm{~h}$ at $37^{\circ} \mathrm{C}$.

A co-immunoprocipitation was performed on the cells, using anti-V5 Agarose Affinity Gel antibody produced in mouse (A7345, MERCK) following the protocol described in the main methods section. EHD4 precipitation was confirmed using EHD4 antibody (1:500, ab153892 Abcam) and co-precipitated ARP-T1 was analyzed using ARP-T1 antiserum (1:2000, GP-SH6).

\section{ACKNOWLEDGEMENTS}

658 We are thankful to Prof. Rune Toftgård ${ }_{2}$ to Prof. Anthony Oro, and to Dr. Sergey Nikolaev for their 659 kind advice. We also acknowledge the Protein Analysis Facility of the University of Lausanne for 660 the mass spectrometry assay. This study was funded by Swiss National Science Foundation 661 (grant 310030-173102), "Fondation Professeur Placide Nicod" and "Fondation Dind Cottier pour 662 la recherche sur la peau". 
664

665

666

\section{AUTHOR CONTRIBUTIONS}

Please see corresponding file.

\section{COMPETING INTERESTS}

Please see the corresponding file.

\section{REFERENCES}

1. Bazex, A., Dupre, A. \& Christol, B. [Follicular atrophoderma, baso-cellular proliferations and hypotrichosis]. Annales de dermatologie et de syphiligraphie 93, 241-254 (1966).

2. Parren, L.J. \& Frank, J. Hereditary tumour syndromes featuring basal cell carcinomas. The British journal of dermatology 165, 30-34 (2011).

3. Vabres, P., et al. The gene for Bazex-Dupre-Christol syndrome maps to chromosome Xq. The Journal of investigative dermatology 105, 87-91 (1995).

4. Abuzahra, F., Parren, L.J. \& Frank, J. Multiple familial and pigmented basal cell carcinomas in early childhood - Bazex-Dupre-Christol syndrome. Journal of the European Academy of Dermatology and Venereology : JEADV 26, 117-121 (2012).

5. Castori, M., Castiglia, D., Passarelli, F. \& Paradisi, M. Bazex-Dupre-Christol syndrome: an ectodermal dysplasia with skin appendage neoplasms. European journal of medical genetics 52, 250-255 (2009).

6. Bal, E., et al. Mutations in ACTRT1 and its enhancer RNA elements lead to aberrant activation of Hedgehog signaling in inherited and sporadic basal cell carcinomas. Nat Med 23, 1226-1233 (2017).

7. Heid, H., et al. Novel actin-related proteins Arp-T1 and Arp-T2 as components of the cytoskeletal calyx of the mammalian sperm head. Experimental cell research 279, 177 187 (2002).

8. Schou, K.B., Pedersen, L.B. \& Christensen, S.T. Ins and outs of GPCR signaling in primary cilia. EMBO Rep 16, 1099-1113 (2015).

9. Liu, H., Kiseleva, A.A. \& Golemis, E.A. Ciliary signalling in cancer. Nat Rev Cancer 18, 511-524 (2018).

10. Briscoe, J. \& Therond, P.P. The mechanisms of Hedgehog signalling and its roles in development and disease. Nature reviews. Molecular cell biology 14, 416-429 (2013).

11. Kasper, M., Jaks, V., Hohl, D. \& Toftgard, R. Basal cell carcinoma - molecular biology and potential new therapies. The Journal of clinical investigation 122, 455-463 (2012).

12. Han, Y.G., et al. Dual and opposing roles of primary cilia in medulloblastoma development. Nat Med 15, 1062-1065 (2009).

13. Wong, S.Y., et al. Primary cilia can both mediate and suppress Hedgehog pathwaydependent tumorigenesis. Nat Med 15, 1055-1061 (2009). 
14. Schmidt, K.N., et al. Cep164 mediates vesicular docking to the mother centriole during early steps of ciliogenesis. J Cell Biol 199, 1083-1101 (2012).

15. Lu, Q., et al. Early steps in primary cilium assembly require EHD1/EHD3-dependent ciliary vesicle formation. Nat Cell Biol 17, 531 (2015).

16. Kim, J., et al. Actin remodelling factors control ciliogenesis by regulating YAP/TAZ activity and vesicle trafficking. Nat Commun 6, 6781 (2015).

17. Reiter, J.F., Blacque, O.E. \& Leroux, M.R. The base of the cilium: roles for transition fibres and the transition zone in ciliary formation, maintenance and compartmentalization. EMBO Rep 13, 608-618 (2012).

18. Roberson, E.C., et al. TMEM231, mutated in orofaciodigital and Meckel syndromes, organizes the ciliary transition zone. J Cell Biol 209, 129-142 (2015).

19. Reiter, J.F. \& Leroux, M.R. Genes and molecular pathways underpinning ciliopathies. Nature reviews. Molecular cell biology 18, 533-547 (2017).

20. Katoh, Y., et al. Practical method for targeted disruption of cilia-related genes by using CRISPR/Cas9-mediated, homology-independent knock-in system. Mol Biol Cell 28, 898906 (2017).

21. Hazim, R.A., Volland, S., Yen, A., Burgess, B.L. \& Williams, D.S. Rapid differentiation of the human RPE cell line, ARPE-19, induced by nicotinamide. Exp Eye Res 179, 18-24 (2018).

22. Bae, J.E., et al. Fine particulate matter (PM2.5) inhibits ciliogenesis by increasing SPRR3 expression via c-Jun activation in RPE cells and skin keratinocytes. Sci Rep 9, 3994 (2019).

23. Pugacheva, E.N., Jablonski, S.A., Hartman, T.R., Henske, E.P. \& Golemis, E.A. HEF1dependent Aurora A activation induces disassembly of the primary cilium. Cell 129, 1351-1363 (2007).

24. Hu, Q., et al. A septin diffusion barrier at the base of the primary cilium maintains ciliary membrane protein distribution. Science 329, 436-439 (2010).

25. Goncalves, J., et al. LUZP1 and the tumor suppressor EPLIN modulate actin stability to restrict primary cilia formation. J Cell Biol 219(2020).

26. Joo, E., Surka, M.C. \& Trimble, W.S. Mammalian SEPT2 is required for scaffolding nonmuscle myosin II and its kinases. Dev Cell 13, 677-690 (2007).

27. Estey, M.P., Di Ciano-Oliveira, C., Froese, C.D., Bejide, M.T. \& Trimble, W.S. Distinct roles of septins in cytokinesis: SEPT9 mediates midbody abscission. J Cell Biol 191, 741-749 (2010).

28. Meng, D., Frank, A.R. \& Jewell, J.L. mTOR signaling in stem and progenitor cells. Development 145(2018).

29. Seo, S., et al. Controlling superconductivity by tunable quantum critical points. Nat Commun 6, 6433 (2015).

30. Nachury, M.V., et al. A core complex of BBS proteins cooperates with the GTPase Rab8 to promote ciliary membrane biogenesis. Cell 129, 1201-1213 (2007). 
31. Aldahmesh, M.A., et al. IFT27, encoding a small GTPase component of IFT particles, is mutated in a consanguineous family with Bardet-Biedl syndrome. Hum Mol Genet 23, 3307-3315 (2014).

32. Cuellar, J., et al. The structure of CCT-Hsc70 NBD suggests a mechanism for Hsp70 delivery of substrates to the chaperonin. Nat Struct Mol Biol 15, 858-864 (2008).

33. Bhowmick, R., et al. Photoreceptor IFT complexes containing chaperones, guanylyl cyclase 1 and rhodopsin. Traffic 10, 648-663 (2009).

34. Takayama, S., Xie, Z. \& Reed, J.C. An evolutionarily conserved family of Hsp70/Hsc70 molecular chaperone regulators. J Biol Chem 274, 781-786 (1999).

35. Zhang, J., Naslavsky, N. \& Caplan, S. Rabs and EHDs: alternate modes for traffic control. Biosci Rep 32, 17-23 (2012).

36. George, M., et al. Ehd4 is required to attain normal prepubertal testis size but dispensable for fertility in male mice. Genesis 48, 328-342 (2010).

37. Tokhtaeva, E., et al. Septin dynamics are essential for exocytosis. J Biol Chem 290, 5280-5297 (2015).

38. Mostowy, S. \& Cossart, P. Septins: the fourth component of the cytoskeleton. Nature reviews. Molecular cell biology 13, 183-194 (2012).

39. Ghossoub, R., et al. Septins 2, 7 and 9 and MAP4 colocalize along the axoneme in the primary cilium and control ciliary length. J Cell Sci 126, 2583-2594 (2013).

40. Muresan, V., Joshi, H.C. \& Besharse, J.C. Gamma-tubulin in differentiated cell types: localization in the vicinity of basal bodies in retinal photoreceptors and ciliated epithelia. $J$ Cell Sci 104 ( Pt 4), 1229-1237 (1993).

41. Benmerah, A. The ciliary pocket. Current opinion in cell biology 25, 78-84 (2013).

42. Malicki, J.J. \& Johnson, C.A. The Cilium: Cellular Antenna and Central Processing Unit. Trends Cell Biol 27, 126-140 (2017).

43. Uddin, B., et al. The human phosphatase CDC14A modulates primary cilium length by regulating centrosomal actin nucleation. EMBO Rep 20(2019).

44. Kim, J., et al. Functional genomic screen for modulators of ciliogenesis and cilium length. Nature 464, 1048-1051 (2010).

45. Hsiao, Y.C., et al. Ahi1, whose human ortholog is mutated in Joubert syndrome, is required for Rab8a localization, ciliogenesis and vesicle trafficking. Hum Mol Genet 18, 3926-3941 (2009).

46. Thoma, C.R., et al. pVHL and GSK3beta are components of a primary ciliummaintenance signalling network. Nat Cell Bio/ 9, 588-595 (2007).

47. Moser, J.J., Fritzler, M.J. \& Rattner, J.B. Primary ciliogenesis defects are associated with human astrocytoma/glioblastoma cells. BMC Cancer 9, 448 (2009).

48. Moser, J.J., Fritzler, M.J. \& Rattner, J.B. Ultrastructural characterization of primary cilia in pathologically characterized human glioblastoma multiforme (GBM) tumors. BMC Clin Pathol 14, 40 (2014). 
49. Miyamoto, T., et al. Insufficiency of BUBR1, a mitotic spindle checkpoint regulator, causes impaired ciliogenesis in vertebrates. Hum Mol Genet 20, 2058-2070 (2011).

50. Fliegauf, M., Kahle, A., Haffner, K. \& Zieger, B. Distinct localization of septin proteins to ciliary sub-compartments in airway epithelial cells. Biol Chem 395, 151-156 (2014).

51. Kukic, I., Rivera-Molina, F. \& Toomre, D. The IN/OUT assay: a new tool to study ciliogenesis. Cilia 5, 23 (2016).

52. Pejskova, P., et al. KIF14 controls ciliogenesis via regulation of Aurora A and is important for Hedgehog signaling. J Cell Biol 219(2020).

53. Sahin, Z., et al. Dynamic Hedgehog signalling pathway activity in germline stem cells. Andrology 2, 267-274 (2014).

54. Makela, J.A., et al. Hedgehog signalling promotes germ cell survival in the rat testis. Reproduction 142, 711-721 (2011).

55. Zhao, X., et al. A Transposon Screen Identifies Loss of Primary Cilia as a Mechanism of Resistance to SMO Inhibitors. Cancer Discov 7, 1436-1449 (2017).

56. Bangs, F. \& Anderson, K.V. Primary Cilia and Mammalian Hedgehog Signaling. Cold Spring Harb Perspect Biol 9(2017).

57. Rack, P.G., et al. Arhgap36-dependent activation of Gli transcription factors. Proc Natl Acad Sci U S A 111, 11061-11066 (2014).

58. Takeshima, H., et al. Frequent involvement of chromatin remodeler alterations in gastric field cancerization. Cancer Lett 357, 328-338 (2015).

59. Patil, P.A., Lombardo, K., Sturtevant, A., Mangray, S. \& Yakirevich, E. Loss of Expression of a Novel Chromatin Remodeler SMARCA1 in Soft Tissue Sarcoma. $J$ Cytol Histol 9(2018).

60. Kuonen, F., et al. Loss of Primary Cilia Drives Switching from Hedgehog to Ras/MAPK Pathway in Resistant Basal Cell Carcinoma. The Journal of investigative dermatology 139, 1439-1448 (2019).

61. Glaessl, A., Hohenlautner, U., Landthaler, M. \& Vogt, T. Sporadic Bazex-Dupre-Christollike syndrome: early onset basal cell carcinoma, hypohidrosis, hypotrichosis, and prominent milia. Dermatol Surg 26, 152-154 (2000).

62. Almeida, S., Ryser, S., Obarzanek-Fojt, M., Hohl, D. \& Huber, M. The TRAF-interacting protein (TRIP) is a regulator of keratinocyte proliferation. The Journal of investigative dermatology 131, 349-357 (2011).

63. Shevchenko, A., Tomas, H., Havlis, J., Olsen, J.V. \& Mann, M. In-gel digestion for mass spectrometric characterization of proteins and proteomes. Nat Protoc 1, 2856-2860 (2006).

64. Nesvizhskii, A.I., Keller, A., Kolker, E. \& Aebersold, R. A statistical model for identifying proteins by tandem mass spectrometry. Anal Chem 75, 4646-4658 (2003).

65. Deutsch, E.W., et al. The ProteomeXchange consortium in 2017: supporting the cultural change in proteomics public data deposition. Nucleic Acids Res 45, D1100-D1106 (2017). 
66. Perez-Riverol, Y., et al. The PRIDE database and related tools and resources in 2019: improving support for quantification data. Nucleic Acids Res 47, D442-D450 (2019).

\section{FIGURES:}

\section{Figure 1. ARP-T1 is expressed during epidermal and epithelial differentiation.}

$\mathbf{a}, \mathbf{c}, \mathbf{e}, \mathbf{g}, \mathrm{mRNA}$ expression of $A C T R T 1$ during differentiation of keratinocytes, $\operatorname{NHEK}(\mathbf{a}, \mathrm{N}=5)$ and $\operatorname{HaCaT}(\mathbf{c}, \mathrm{N}=3)$, and epithelial cells, ARPE19 (e, N=3) and hTERT-RPE1 (g, N=5). Data are presented as means of the fold change compared to the value of undifferentiated samples. Each open circle represents one independent experiment. $\mathbf{b}, \mathbf{d}, \mathbf{f}, \mathbf{h}$, Representative images of ARP-T1 expression during differentiation of keratinocytes, NHEK (b) and $\mathrm{HaCaT}(\mathbf{d})$, and epithelial cells, ARPE19 (f) and hTERT-RPE1 (h). ARP-T1 was detected using guinea pig anti-ARP-T1 antisera (b) or mouse antibody $(\mathbf{d}, \mathbf{f}, \mathbf{h}) .{ }^{*}$ indicates polymers of ARP-T1 confirmed by mass spectrometry analysis. Keratin 10 and IFT88 were used as markers of cell differentiation in keratinocytes and epithelial cells respectively, actin and tubulin as loading controls.

\section{Figure 2. ACTRT1 is regulated by non-canonical Hedgehog signaling pathway and by Protein Kinase $\mathbf{C}$ delta.}

$\mathbf{a}, \mathbf{b}$, Relative ACTRT1, GLI1 and PTCH1 mRNA expression (a, $\mathbf{b}, \mathrm{N}=6)$ and ARP-T1 expression (a, $\mathrm{N}=4 ; \mathbf{b}, \mathrm{N}=3$ ) upon treatment with SAG in hTERT-RPE1 cells under proliferative (a) and differentiating (b) conditions. Actin is used as loading control for ARP-T1 expression. Numbers under the blots present the fold change expression of ARP-T1 compared to the vehicle (DMSO) treatment. c-e Relative ACTRT1 (c, N=7), GLI1 (d, N=4), PTCH1 (e, N=3) mRNA expression and ARP-T1 expression (c, N=4) upon treatment with purmorphamine (PUR) and/or vismodegib (VISMO) in differentiated hTERT-RPE1 cells. Tubulin is used as loading control for ARP-T1 expression. Numbers under the blots present the fold change expression of ARP-T1 compared to the vehicle treatment. $\mathbf{f}, \mathbf{g}$, Relative ACTRT1 mRNA expression upon treatment with different protein kinase inhibitors $(\mathbf{f})$ or with PKC inhibitors $(\mathbf{g})$ in differentiated NHEK $(\mathrm{N}=3)$. $\mathbf{h}$, Relative ACTRT1 mRNA expression upon treatment with PKC inhibitors in differentiated HaCaT cells $(\mathrm{N}=3)$. a-h, Data are presented as means of the fold change compared to the value of vehicle treated samples. Each open circle represents one independent experiment. i, Schematic representation of ARP-T1 and ARP-T1 mutant (547_548insA) with predicted phosphorylation sites. j, ARP-T1 and Phospho-Serine (P-Ser) PKC expression in NHEK transduced with lentiviral vectors, empty vector (V), ACTRT1 mutant (M) and ACTRT1 WT (WT), after immunoprecipitated (IP) with anti-FLAG monoclonal antibody M2-conjugated agarose.

\section{Figure 3. ARP-T1 interacts with proteins involved in ciliary machinery.}

a,b, HeLa (a) and hTERT-RPE1 (b) cells were transduced with lentiviral vectors, empty vector (V), ACTRT1 mutant (M) and ACTRT1 WT (WT), and immunoprecipitated (IP) with anti-FLAG monoclonal antibody M2-conjugated agarose, and analyzed by immuno-blot with indicated antisera. c, Immunofluorescence stainings of ARP-T1, acetylated-tubulin and rootletin in 35 days of serum-starved ARPE19 cells. Nuclei are stained with DAPI. Scale bar, $5 \mu \mathrm{m}$. Higher magnifications of the boxed area are shown on right three panels. Scale bar, $1 \mu \mathrm{m}$. d, Immunofluorescence staining of ARP-T1, gamma-tubulin, EHD4, and septin 2 in 48 h of serumstarved hTERT-RPE1 cells. Nuclei are stained with DAPI. Scale bar, $5 \mu \mathrm{m}$. Higher magnifications of the boxed area are shown on the right three panels. Scale bar, $1 \mu \mathrm{m}$. 
867

868

869

870

871

872

873

874

875

876

877

878

879

880

881

882

883

884

885

886

887

888

889

890

891

892

893

894

895

896

897

898

899

900

901

902

903

904

905

Figure 4. The Bazex-Dupré-Christol syndrome is a ciliopathy caused by ARP-T1 loss of function, and knock-down of ACTRT1 in hTERT-RPE1 cells induces resorption of primary cilia.

a, Representative immunofluorescence images using acetylated-tubulin (green) and rootletin (red) (left), and ARP-T1 (green) and rootletin (red) (right) in hair follicle, sporadic BCC and 4 BDCS (ACTRT1547_548insA, mutation B2, mutation A3, mutation $\mathrm{CNE} 12^{6}$ ). Cell nuclei are stained with DAPI (blue). Scale bar, $5 \mu \mathrm{m}$. b, Quantification of the ciliary length from 3D confocal immunofluorescence microscopy images. c,d, Quantification of the relative fluorescence intensity of rootletin (c, N=5) and ARP-T1 $(\mathbf{d}, \mathrm{N}=10)$ on the ciliary rootlet. e,f, Correlation between the ARPT1 fluorescence and ciliary length (e), and between the ARP-T1 and rootletin fluorescence (f). $\mathbf{g}$. Immunofluorescence stainings of acetylated-tubulin (green) and rootletin (pink) in $48 \mathrm{~h}$ serum starved hTERT-RPE1 cells- expressing an empty vector (V), or ACTRT1 mutant (M), or ACTRT1 WT (WT). Cell nuclei are stained with DAPI (blue). Scale bar, $10 \mu \mathrm{m} . \mathbf{h}$, Quantification of ciliary length of g. i, Immunofluorescence stainings of acetylated-tubulin (green) and rootletin (pink) in $48 \mathrm{~h}$ serum-starved control and ACTRT1 KD hTERT-RPE1 cells. Cell nuclei are stained with DAPI (blue). Scale bar, $10 \mu \mathrm{m}$. j,k, Quantification of the ciliary length (j) and ARP-T1 protein level (k, $\mathrm{N}=3$ ) in control (Cont.) and ACTRT1 KD hTERT-RPE1 cells. I, Quantification of ciliary length in 48 h serum-starved control and ACTRT1 KD hTERT-RPE1 cells expressing an empty vector (V), or ACTRT1 mutant resistant to shRNA (MshR). b-d,h,j,l, Results are presented as Tukey box-plot. Black circles represent outliers. $\mathbf{m}$, Representative immunofluorescence stainings of ARP-T1 (pink) and rootletin (green, top) or septin 2 (green, bottom) upon treatment with SAG or purmorphamine (PUR) in hTERT-RPE1 cells under differentiating condition. Scale bar, $5 \mu \mathrm{m}$ (top) or $1 \mu \mathrm{m}$ (bottom). $\mathbf{n}$, Immunofluorescence stainings of acetylated-tubulin (green) and septin 9 (pink, top) or septin 2 (pink, bottom) in $48 \mathrm{~h}$ serum-starved control and ACTRT1 KD hTERT-RPE1 cells. Scale bar, $1 \mu \mathrm{m} . \mathbf{0}$, Percentage of ciliated control and ACTRT1 KD hTERT-RPE1 cells under proliferative condition, after treatment with cytochalasin D (CytD). Data are presented as means of the percentage $+/$-SD.

\section{Figure 5. ARP-T1 localizes to midbody during cytokinesis}

a, Immunofluorescence stainings of ARP-T1 (red) and acetylated-tubulin (green) in hTERTRPE1 cells. Cell nuclei are stained with DAPI (blue). b, Immunofluorescence stainings of ARPT1 (green) and acetylated-tubulin (red) in HeLa cells. Cell nuclei are stained with DAPI (blue). c, Immunofluorescence stainings of ARP-T1 (red) and acetylated-tubulin (green) in HaCaT cells. Cell nuclei are stained with DAPI (blue). $\underline{\mathbf{d}, \mathbf{e}, \text { Proliferation (d) and apoptosis (e) analyses of }}$ control (Cont.) and ACTRT1 KD hTERT-RPE1 cells. Data are presented as means of the percentage. Each open circle represents one independent experiment. f-i, Model for ARP-T1 travelling from midbody to the PC. 